\title{
Cumulative short-lived photofission product yields for nuclear forensic application
}

\section{July 2021}

Ariana A Foley, Haori Yang

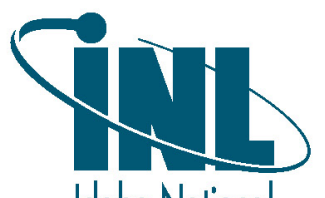

Idaho National

Laboratory 


\section{DISCLAIMER}

This information was prepared as an account of work sponsored by an agency of the U.S. Government. Neither the U.S. Government nor any agency thereof, nor any of their employees, makes any warranty, expressed or implied, or assumes any legal liability or responsibility for the accuracy, completeness, or usefulness, of any information, apparatus, product, or process disclosed, or represents that its use would not infringe privately owned rights. References herein to any specific commercial product, process, or service by trade name, trade mark, manufacturer, or otherwise, does not necessarily constitute or imply its endorsement, recommendation, or favoring by the U.S. Government or any agency thereof. The views and opinions of authors expressed herein do not necessarily state or reflect those of the U.S. Government or any agency thereof. 


\section{Cumulative short-lived photofission product yields for nuclear forensic application}

Ariana A Foley, Haori Yang

July 2021

Idaho National Laboratory

Idaho Falls, Idaho 83415

http://www.inl.gov

Prepared for the U.S. Department of Energy Under DOE Idaho Operations Office

Contract DE-AC07-05ID14517 


\section{ANS Virtual}

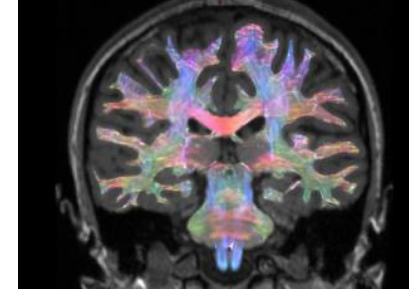

Nuclear is good for you.

Winter Meeting

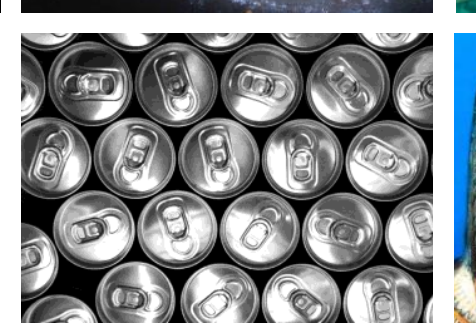

Cumulative short-lived photofission

$=\frac{1}{2}$ product yields for nuclear forensic application

\section{Ari Foley ${ }^{1,2}$ and Haori Yang ${ }^{2}$}

${ }^{1}$ Idaho National Laboratory, Idaho Falls, ID 83415

2 School of Nuclear Science \& Engineering, Oregon State University, Corvallis, OR 97333
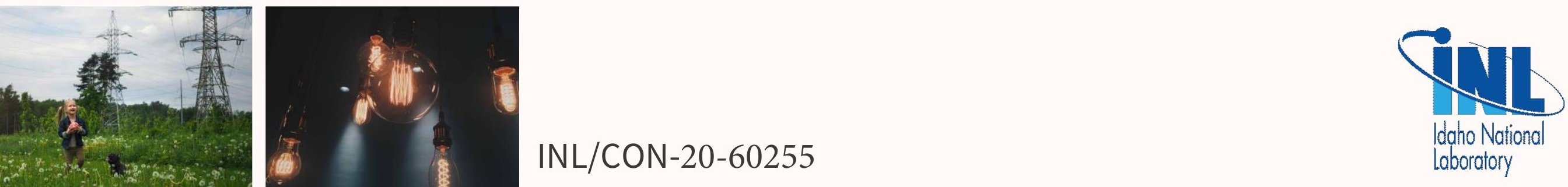

Oregon State University 


\section{Introduction}

- In post-detonation nuclear forensics, FPY distribution could give indication to the neutron energy spectrum that had induced fission

- Photon-induced fission, "photofission", is most probable at GDR and the resulting FPY distribution around this energy closely resembles that of DT neutron fission

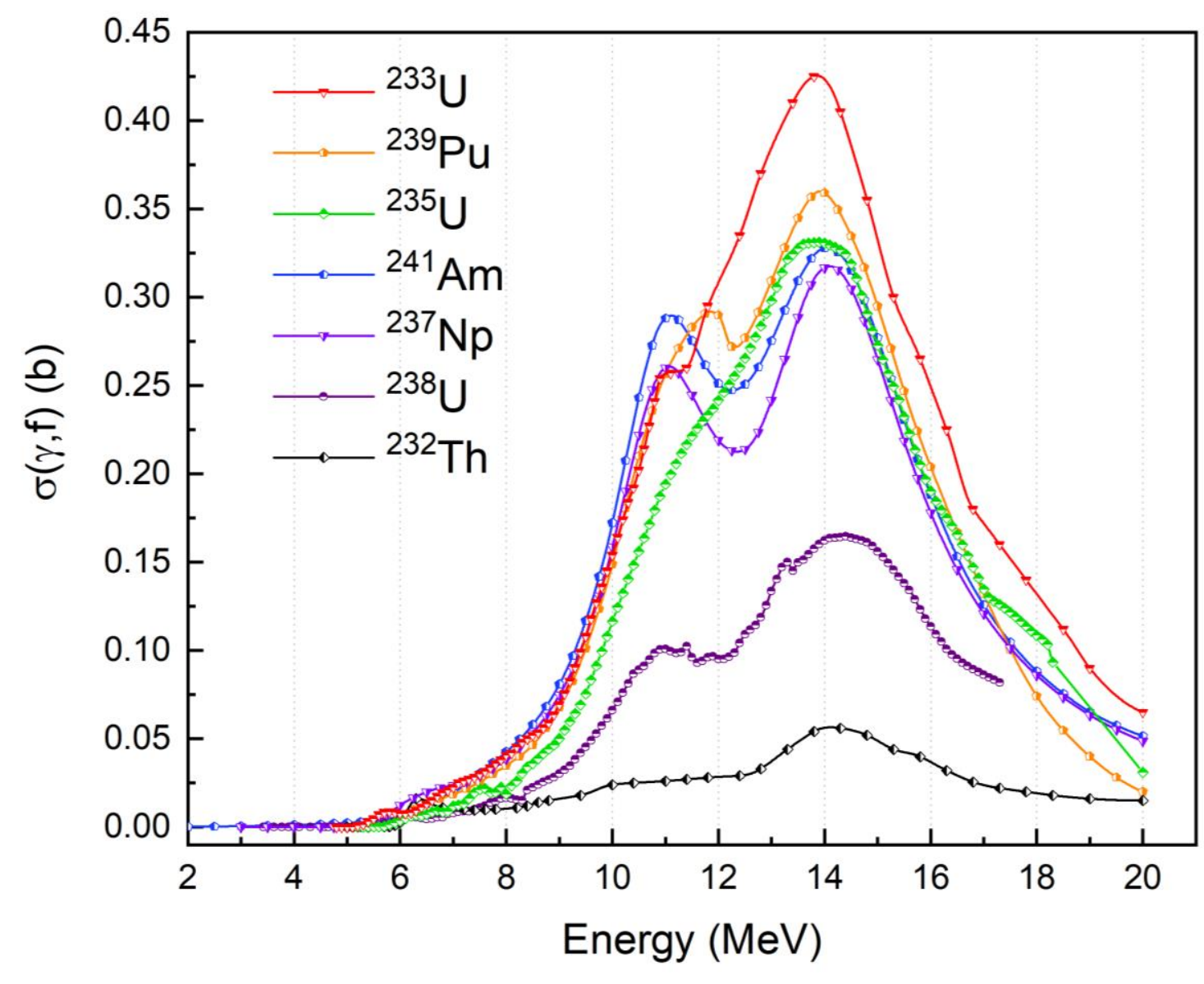

${ }^{1}$ ENDF-B/VII.0: Accessed from Janis 4.0, Nuclear Energy Agency, Organization for Economic Co-operation and Development. https://www.oecd-nea.org/janis/ 


\section{Motivation}

\section{- Improving nuclear data}

- Photonuclear data heavily reliant on nuclear models; limited experimental data often inconsistent

- New and improved isotope production for isotopes of interest to nuclear forensics

- Less costly and more flexible method to benefit nuclear forensics exercises allowing analysis techniques to be tested 


\section{Objectives}

1. Measure short lived $\left(\mathrm{T}_{1 / 2} \leq 50 \mathrm{~s}\right)$ photofission product yields for ${ }^{238} \mathrm{U}$ and ${ }^{232} \mathrm{Th}$ at 8,14 , and $20 \mathrm{MeV}$ endpoint energies

2. Perform thorough uncertainty analysis to ensure uncertainty can be confidently declared

3. Develop experimental and analysis methods for consistent future photofission product yield measurements of additional fissional nuclides and endpoint energies 


\section{Fission Product Yield Distributions}

- Distribution of fission products dependent on several factors; most strongly target nucleus and excitation energy

- In the multimode-fission model, FPY mass distribution is understood by the sum of multiple fission modes

- Symmetric superlong mode (SL), and two asymmetric modes standard I (STI) and standard II (STII) for 3 mode fit (5 Gaussians)

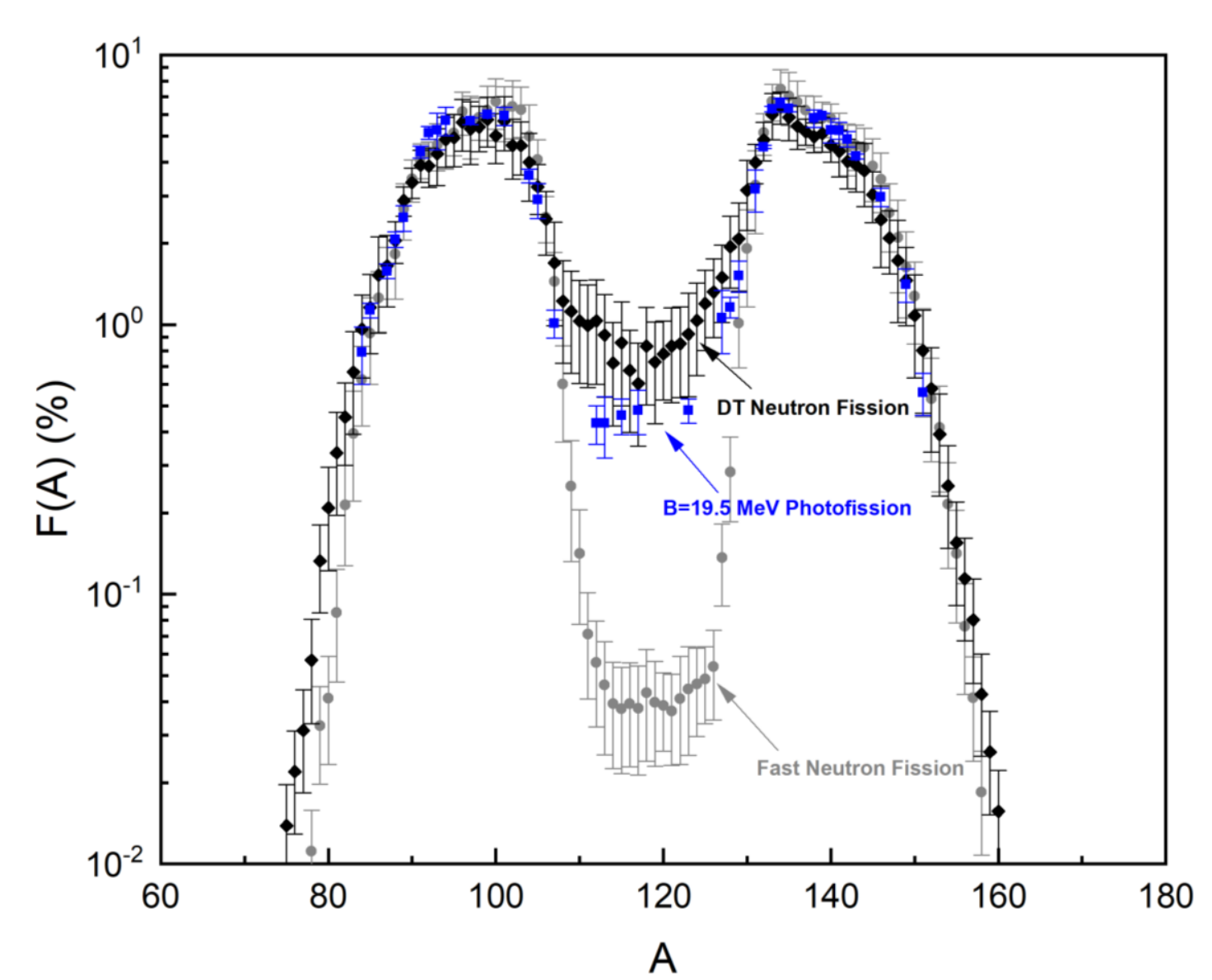

$Y(A)=K_{S L} \cdot e^{-\frac{\left(A-\bar{A}_{S L}\right)^{2}}{2 \sigma^{2} S L}}+K_{S T I} \cdot e^{-\frac{\left(A-\bar{A}_{S L}-D_{S T I}\right)^{2}}{2 \sigma_{S T I}^{2}}}+K_{S T I} \cdot e^{-\frac{\left(A-\bar{A}_{S L}+D_{S T I}\right)^{2}}{2 \sigma_{S T I}^{2}}}+K_{S T I I} \cdot e^{-\frac{\left(A-\bar{A}_{S L}-D_{S T I I}\right)^{2}}{2 \sigma^{2} S T I I}}+K_{S T I I} \cdot e^{-\frac{\left(A-\bar{A}_{S L}+D_{S T I I}\right)^{2}}{2 \sigma^{2}} !}$

${ }^{1}$ ENDF-B/VII.0: Accessed from Janis 4.0, Nuclear Energy Agency, Organization for Economic Co-operation and Development. https://www.oecd-nea.org/janis/

${ }_{2}^{2}$ S.S. Beleshev et al. "Mass yield distributions and fission modes in photofission of ${ }^{238} \mathrm{U}$ below $20 \mathrm{MeV}$ ”. Physical Review C 91, 034603 (2015) 


\section{Multimode Fission Model - 238U}
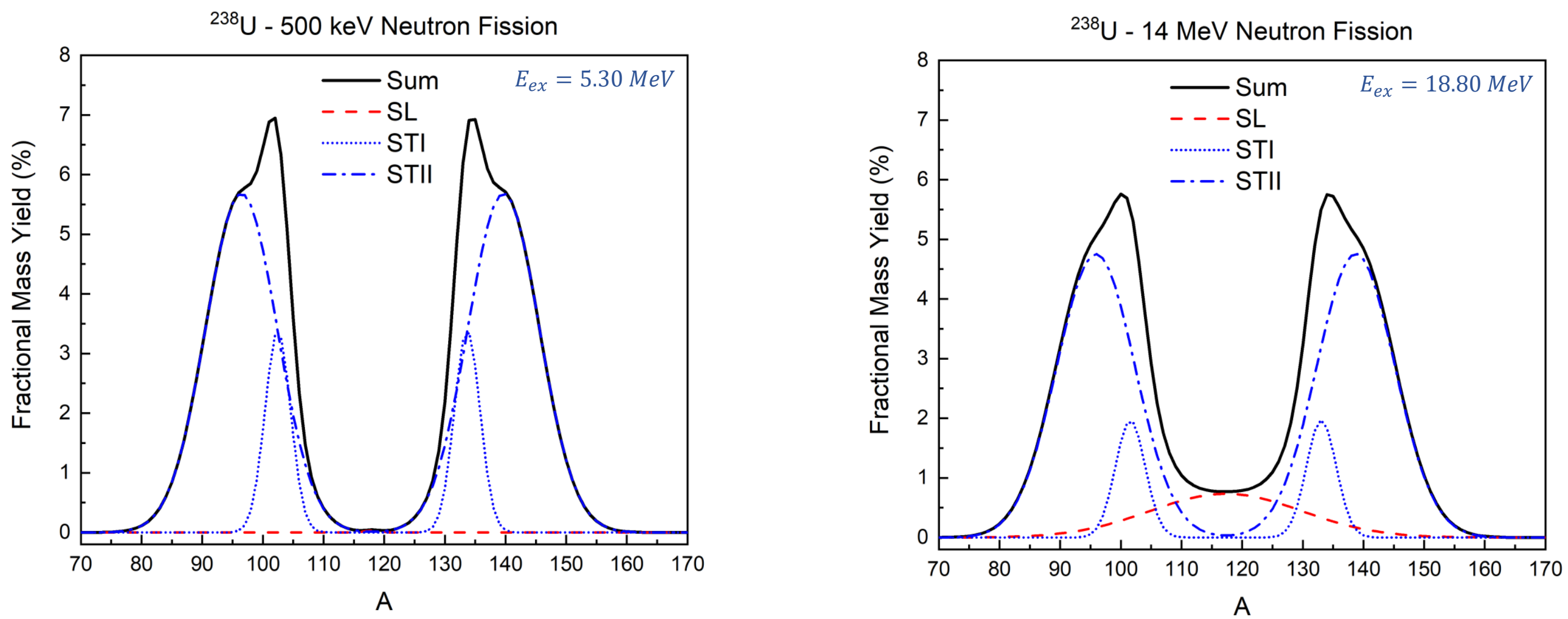

Neutron-induced fission for fast (500 keV) and DT (14 MeV) neutrons used as reference ${ }^{1}$ Symmetric SL mode increases at higher energies

Ratio between two asymmetric STI and STII modes of interest

${ }^{1}$ ENDF-B/VII.0: Accessed from Janis 4.0, Nuclear Energy Agency, Organization for Economic Co-operation and Development. https://www.oecd-nea.org/janis/ 


\section{Multimode Fission Model - 232Th}
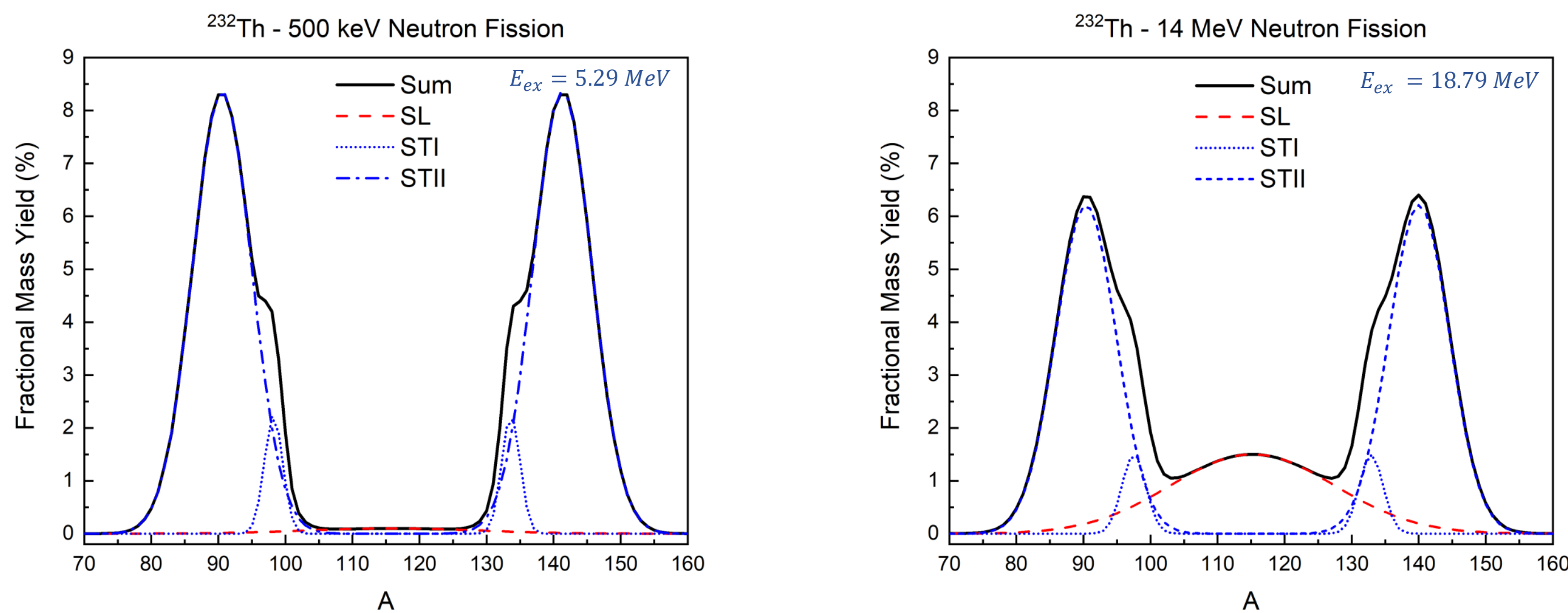

Neutron-induced fission for fast (500 keV) and DT (14 MeV) neutrons used as reference ${ }^{1}$ Third "peak" in valley

Symmetric SL mode increases at higher energies

Difference in two asymmetric STI and STII modes of interest

${ }^{1}$ ENDF-B/VII.0: Accessed from Janis 4.0, Nuclear Energy Agency, Organization for Economic Co-operation and Development. https://www.oecd-nea.org/janis/ 


\section{Experimental Materials and Methods}

ITI Idaho National Laboratory Oregon State 


\section{Photonuclear Reactions}

- Yield of photonuclear reactions calculated with bremsstrahlung X-ray flux and interaction cross sections ${ }^{1}$ necessary to calculate

$$
Y(B)=N_{T} \int_{0}^{B} \frac{\phi(B, E)}{d E} \cdot \sigma_{\gamma, f}(E) d E
$$

- Excitation energy of target nucleus useful in comparing monoenergetic interaction with those produced with energy spectrum

$$
\left\langle E^{*}(B)\right\rangle=\frac{\int_{0}^{B} \frac{\phi(B, E)}{d E} \cdot \sigma_{\gamma, f}(E) E d E}{\int_{0}^{B} \frac{\phi(B, E)}{d E} \cdot \sigma_{\gamma, f}(E) d E}
$$

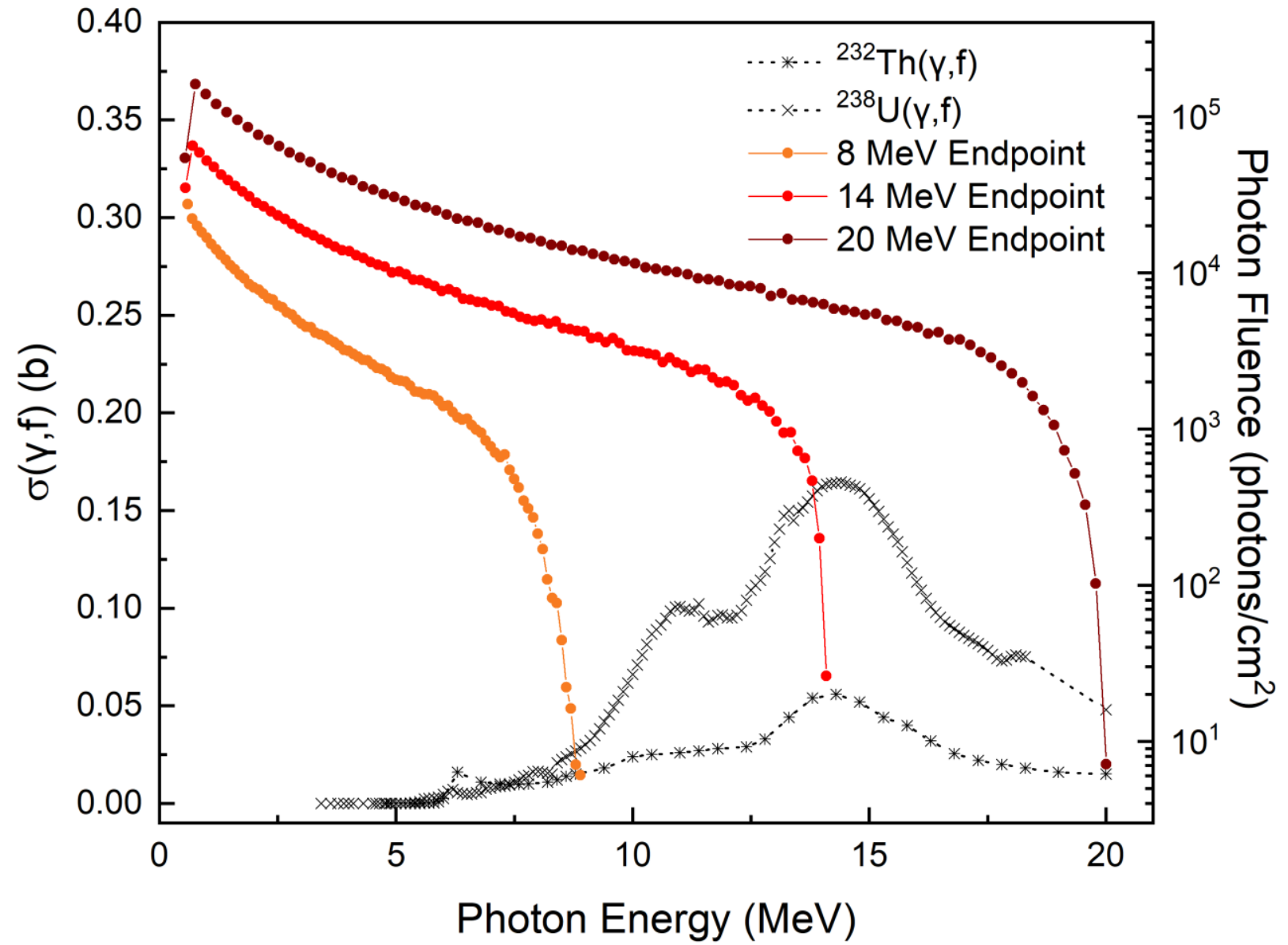

${ }^{1}$ ENDF-B/VII.0: Accessed from Janis 4.0, Nuclear Energy Agency, Organization for Economic Co-operation and Development. https://www.oecd-nea.org/janis/ 


\section{Target Foils}

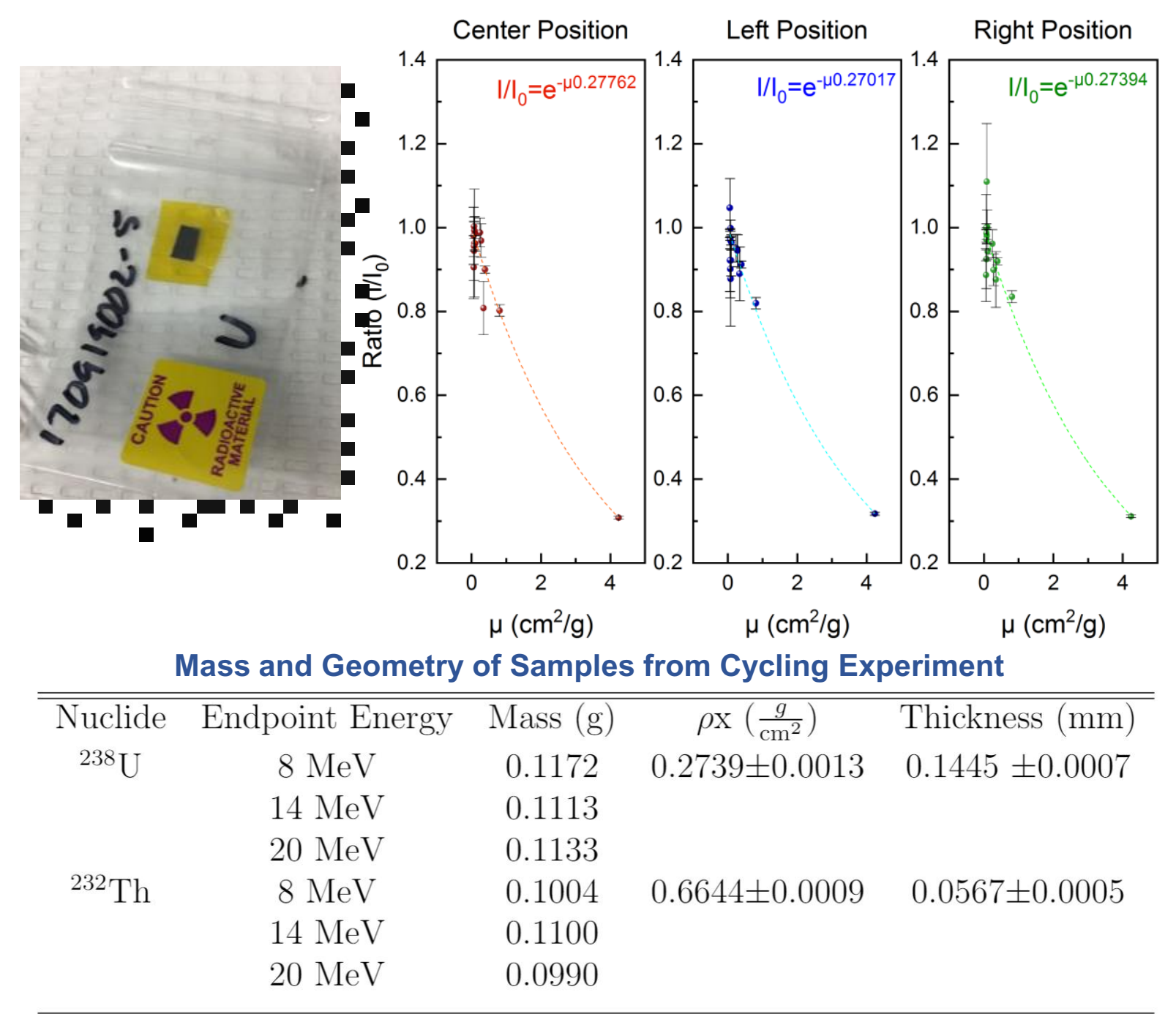

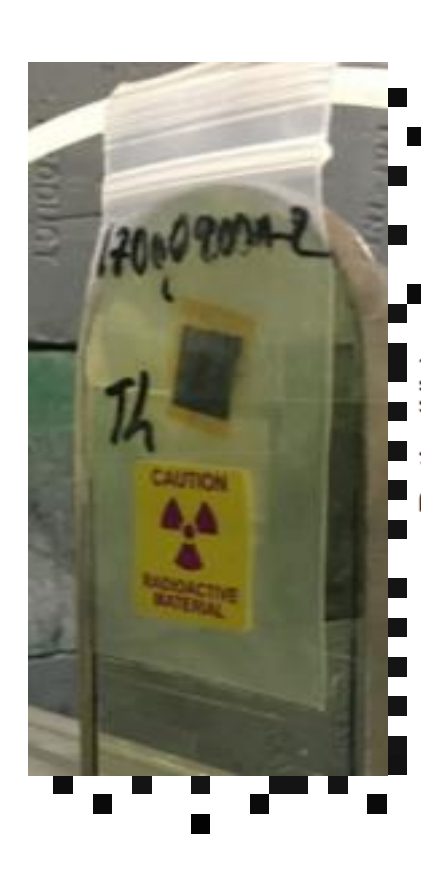

$I / I_{0}=e^{-\mu \rho x}$
Thorium Foil Attenuation Fit

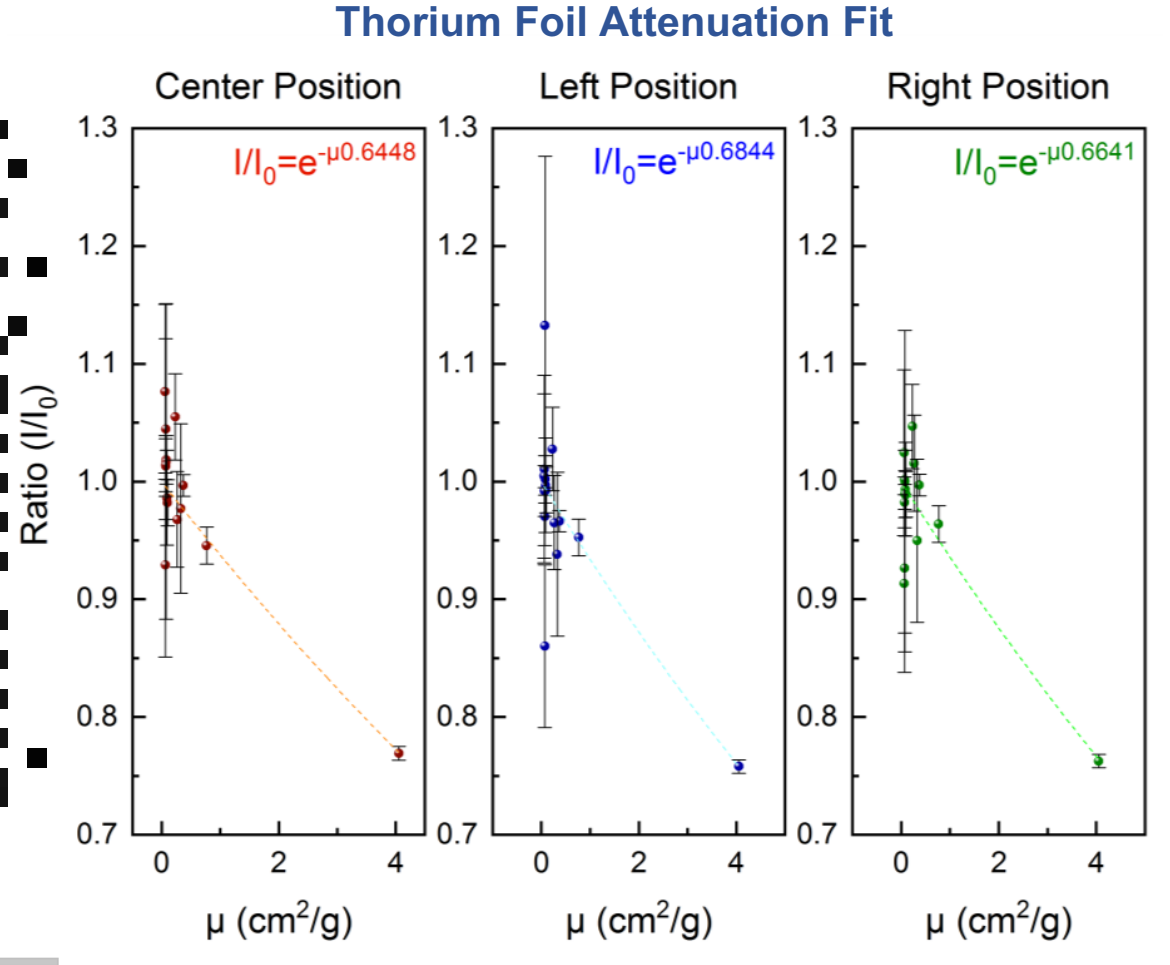

- Foil thickness calculated from ${ }^{152} \mathrm{Eu} y$-ray attenuation

- Attenuation data accessed from NIST XCOM ${ }^{2}$

${ }^{2}$ NIST Physical Measurement Laboratory, XCOM: Photon Cross Sections Database. https://www.nist.gov/pml/xcom-photon-cross-sections-database 


\section{ISU-IAC 25-MeV S-Band Linear Accelerator}

- Experiments performed at the Idaho Accelerator Center (IAC)

- Adjusted Parameters

- Endpoint Energy (B)

- Repetition Rate (in Hz)

- Pulse Width (in $\mu \mathrm{s}$ )

- Measured Parameters

- Charge per pulse (nC)

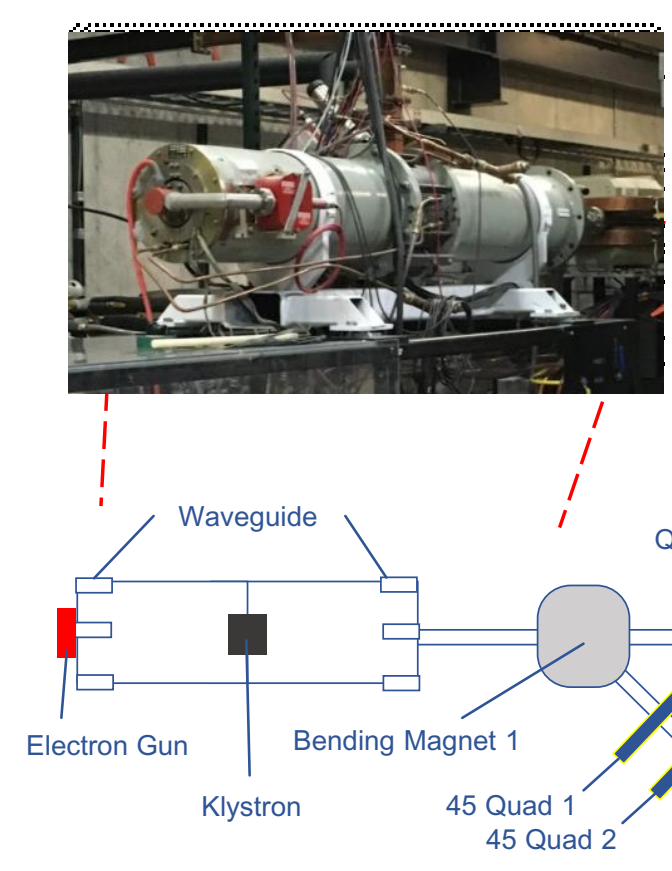

Linac Parameters from Cycling Experiment

\begin{tabular}{cccccc}
\hline \hline Target & $\mathrm{B}(\mathrm{MeV})$ & $\left\langle E^{*}(B)\right\rangle(\mathrm{MeV})$ & $1 / \tau(\mathrm{Hz})$ & Cycles & $Q_{\text {Average }}(\mathrm{nC})$ \\
${ }^{238} \mathrm{U}$ & 8 & $6.78 \pm 0.01$ & 200 & 50 & $361.39 \pm 2.88$ \\
${ }^{238} \mathrm{U}$ & 14 & $10.30 \pm 0.01$ & 15 & 50 & $186.73 \pm 1.26$ \\
${ }^{238} \mathrm{U}$ & 20 & $12.59 \pm 0.01$ & 2 & 50 & $302.42 \pm 1.20$ \\
${ }^{232} \mathrm{Th}$ & 8 & $6.74 \pm 0.02$ & 200 & 60 & $257.90 \pm 1.22$ \\
${ }^{232} \mathrm{Th}$ & 14 & $9.22 \pm 0.01$ & 30 & 60 & $190.01 \pm 1.35$ \\
${ }^{232} \mathrm{Th}$ & 20 & $11.50 \pm 0.03$ & 6 & 50 & $303.36 \pm 1.64$ \\
\hline
\end{tabular}

IIdaho National Laboratory OregonState

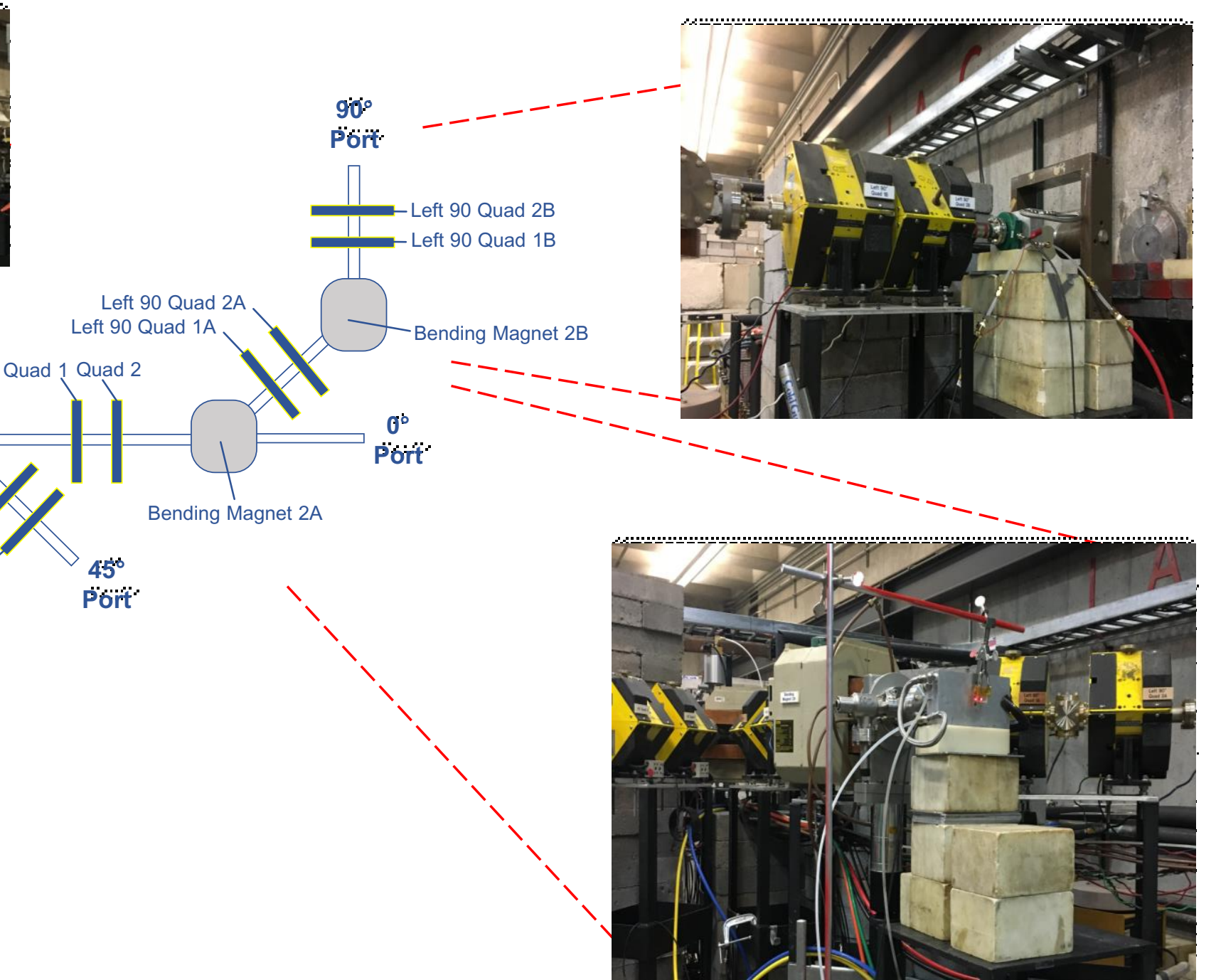




\section{In-Hall Experimental Setup}

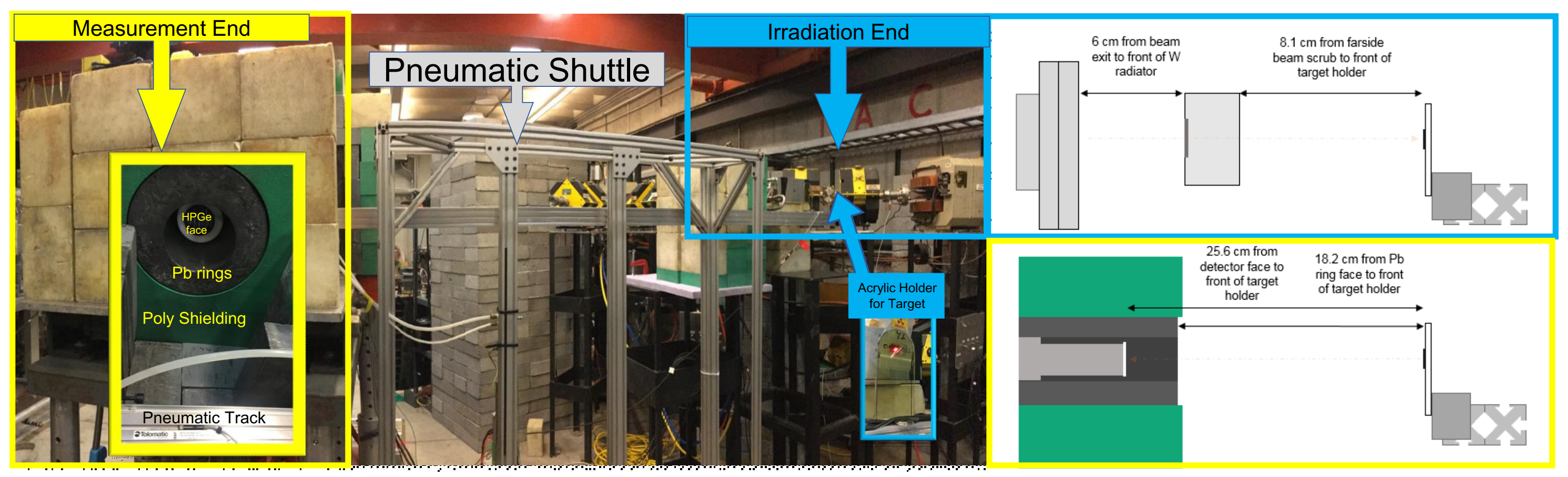

O1. Idaho National Laboratory Oregon State 


\section{Simulation \& Characterization}




\section{Electron Beam to Bremsstrahlung}
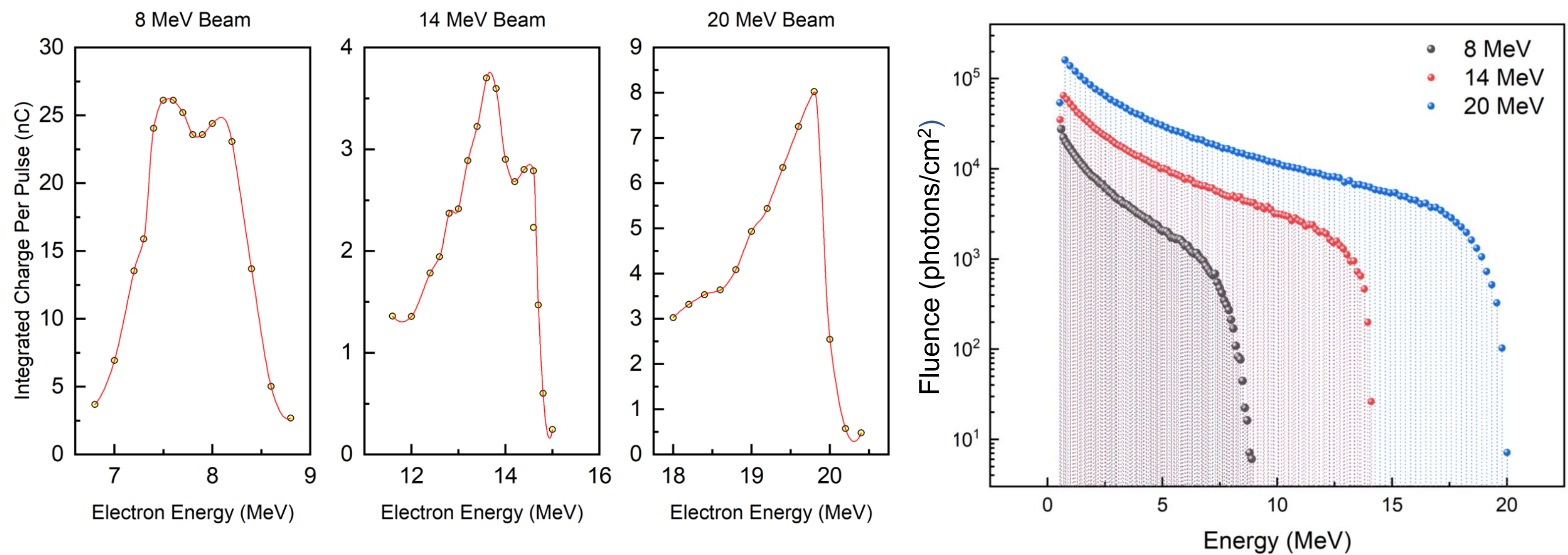

- MCNP6 model of linac end of irradiations to include linac geometry, radiator, beam scrub, current loop, target

- SDEF electron source using experimentally measured electron energy distribution and 200 point Akima spline fit

- Bremsstrahlung X-ray spectra needed in calculation of photofission events per pulse 


\section{Intensity Distribution of Beam on Target}
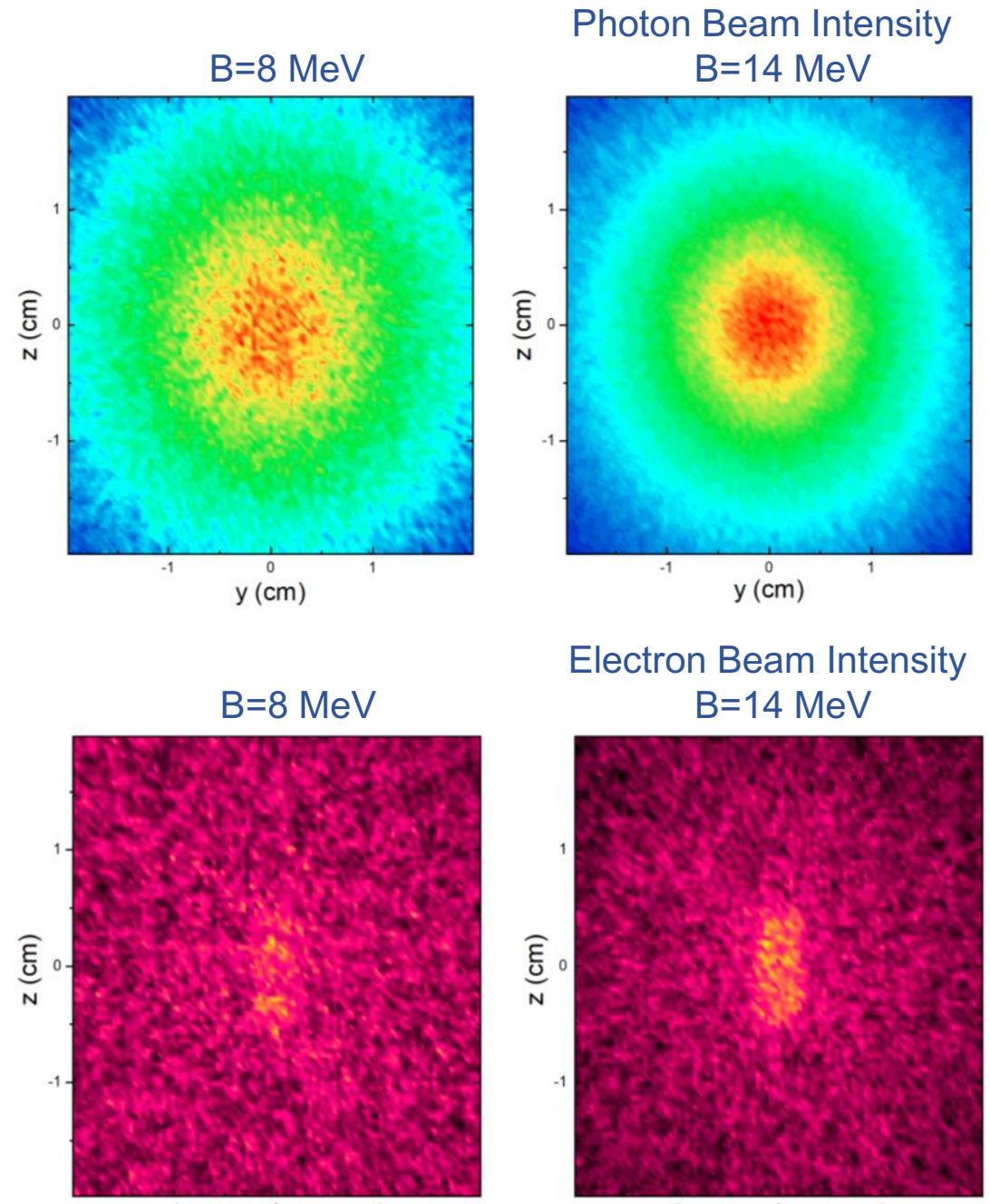

$y \stackrel{0}{(\mathrm{~cm})}$

Electron Beam Intensity

$\mathrm{B}=14 \mathrm{MeV}$

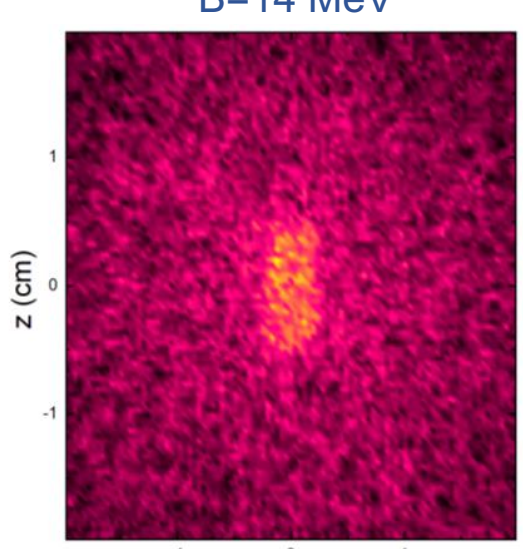

$y(\mathrm{~cm})$

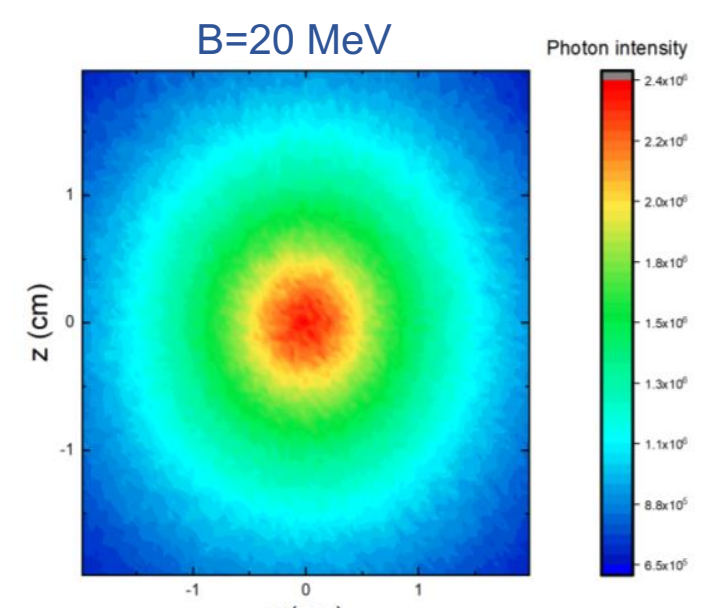

$y(\mathrm{~cm})$
$\mathrm{B}=20 \mathrm{MeV}$

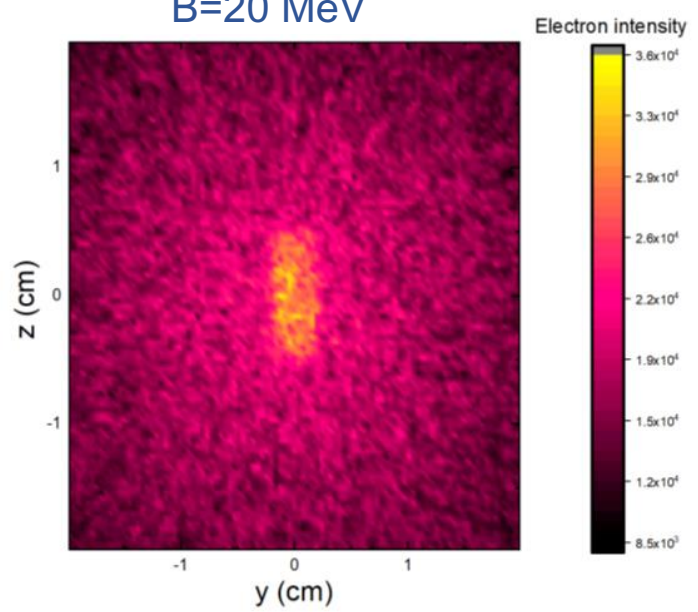

- Mesh tally of photon and electron distribution on target performed in MCNP6

- Visualize the spread of the beam and how photon beam is not uniform over target

- Electron tally shows placement of uranium foil from electron producing interactions

- Experimentally, "center" is determined from irradiation glass and aligning a laser 


\section{Uncertainty Contribution from Neutron Fission}
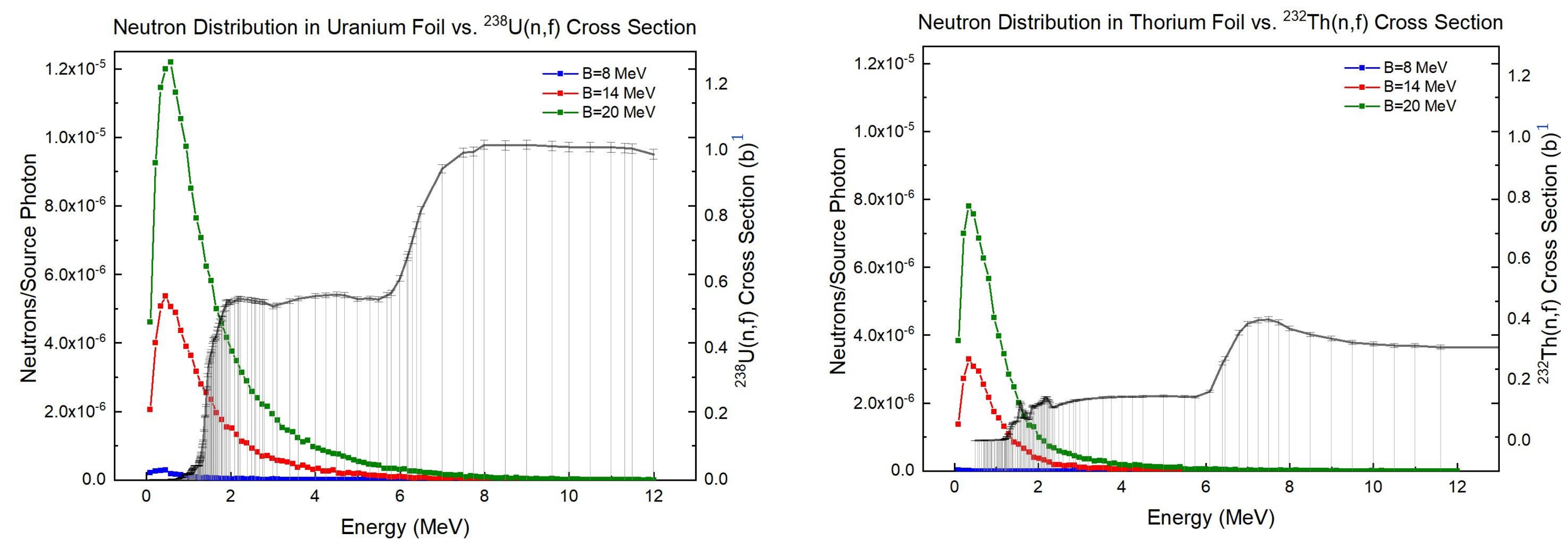

- Neutron flux on targets determined using MCNP6 simulation and ENDF-B/VII.0 neutron fission $\sigma{ }^{1}$

- Negligible contribution from neutron fission verified

${ }^{1}$ ENDF-B/VII.0: Accessed from Janis 4.0, Nuclear Energy Agency, Organization for Economic Co-operation and Development. https://www.oecd-nea.org/janis/ 


\section{Uncertainty Contribution from Impurity Actinides}

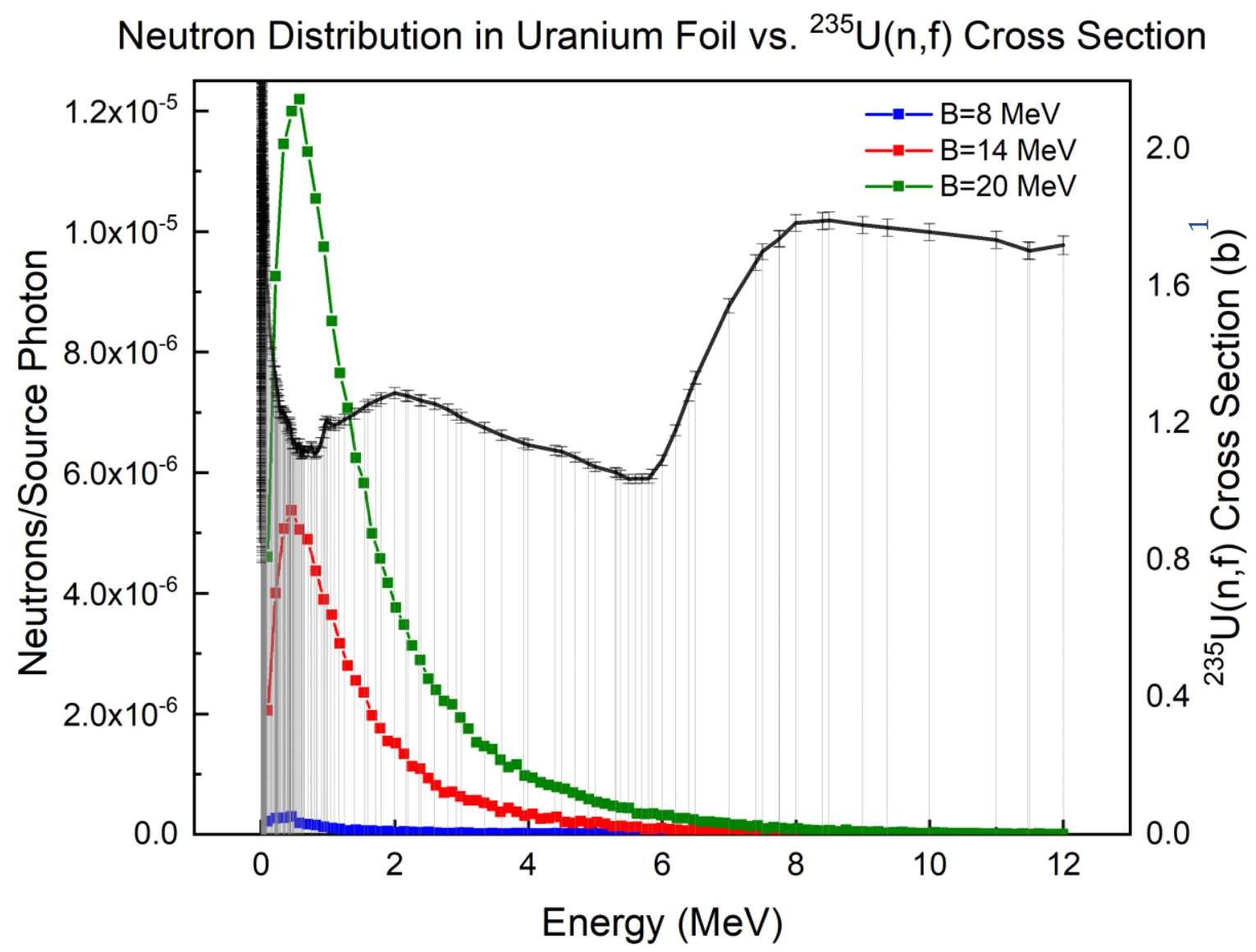

${ }^{1}$ ENDF-B/VII.0: Accessed from Janis 4.0, Nuclear Energy Agency, Organization for Economic Co-operation and Development. https://www.oecd-nea.org/janis/
- ICP-MS isotopic and elemental analysis performed on samples of the uranium and thorium foils at INL

- Uranium matches natural $U$ standard with $A=234$ and 235

- Virtually no impurities of other thorium isotopes

- Photofission and neutron fission yield from ${ }^{235} \mathrm{U}$ impurity in uranium foil, negligible contribution

ICP-MS Isotopic Analysis of Uranium Foil

\begin{tabular}{cccc}
\hline \hline Sample & ${ }^{234} \mathrm{U}($ at. $\%)$ & ${ }^{235} \mathrm{U}($ at. $\%)$ & ${ }^{238} \mathrm{U}($ at. $\%)$ \\
U Foil & $0.0054 \% \pm 0.0002 \%$ & $0.736 \% \pm 0.009 \%$ & $99.26 \% \pm 0.01 \%$ \\
Natural U Standard & $0.0055 \% \pm 0.0002 \%$ & $0.720 \% \pm 0.005 \%$ & $99.27 \% \pm 0.01 \%$
\end{tabular}

ICP-MS Isotopic Analysis of Thorium Foil

\begin{tabular}{ccccc}
\hline \hline Sample & ${ }^{228} \operatorname{Th}($ at.\%) & ${ }^{229} \operatorname{Th}($ at.\%) & ${ }^{230} \operatorname{Th}(a t . \%)$ & ${ }^{232} \operatorname{Th}(a t . \%)$ \\
Th Foil & $<1 \cdot 10^{-4 \%}$ & $<1 \cdot 10^{-4 \%}$ & $<1 \cdot 10^{-4 \%}$ & $99.99992 \% \pm 0.00002 \%$ \\
\hline
\end{tabular}




\section{Fission Product Yield Analysis}




\section{Pulsing and Cycling Structure}

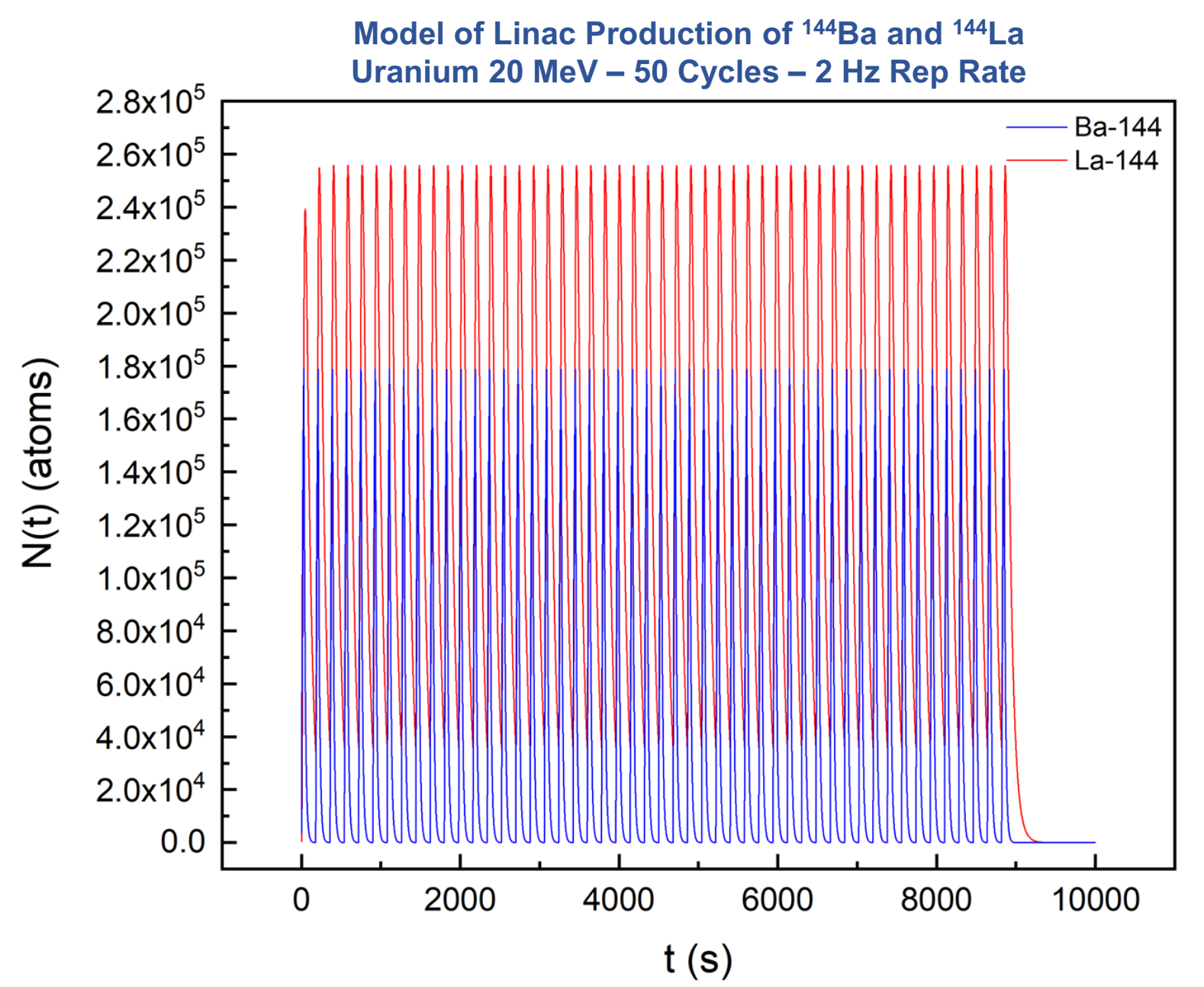

- Pulsing of the accelerator leads to rapid buildup and decay during 30 second irradiation followed by measurement period

- To increase statistics, cycles of irradiation and measurement performed

- Measurements from in between each accelerator pulse summed

- Back-out per pulse contribution considering pulse and cycles

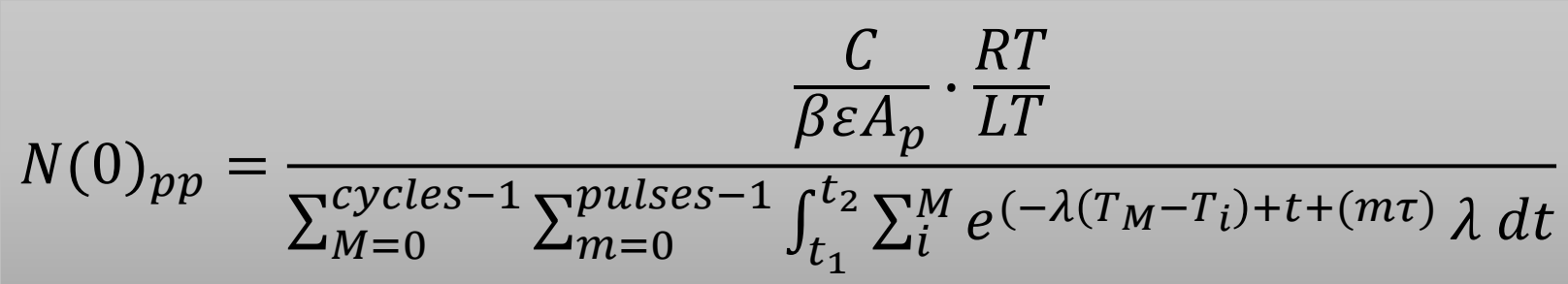

$$
{ }^{144} \mathrm{Ba}\left(\mathrm{T}_{1 / 2}=11.4 \mathrm{~s}\right) \rightarrow{ }^{144} \mathrm{La}\left(\mathrm{T}_{1 / 2}=40.7 \mathrm{~s}\right)
$$




\section{Temporal gamma ray spectroscopy}

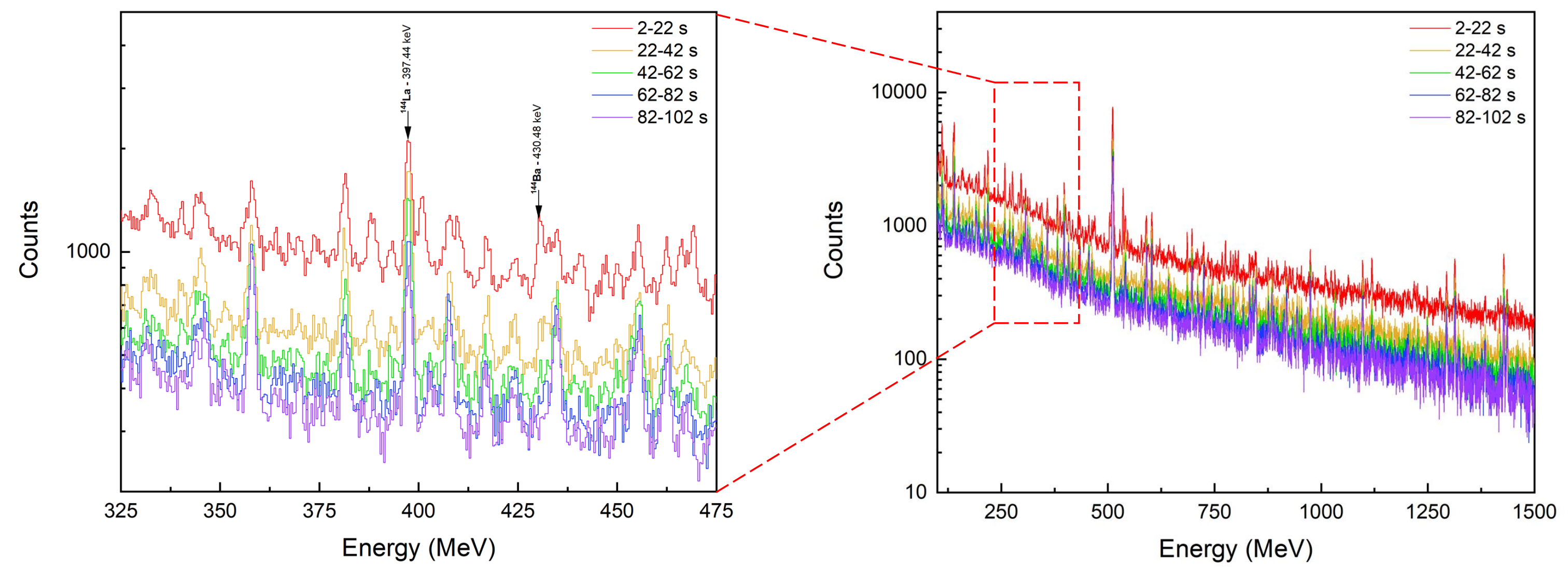

${ }^{144} \mathrm{Ba}\left(\mathrm{T}_{1 / 2}=11.4 \mathrm{~s}\right) \rightarrow{ }^{144} \mathrm{La}\left(\mathrm{T}_{1 / 2}=40.7 \mathrm{~s}\right)$

$$
R(t)=\frac{C}{\beta \varepsilon A_{p}} \frac{R T}{L T}=\int_{x-\frac{\Delta b}{2}}^{x+\frac{\Delta b}{2}} A(t) d E
$$




\section{Integral Decay Rate Fit}
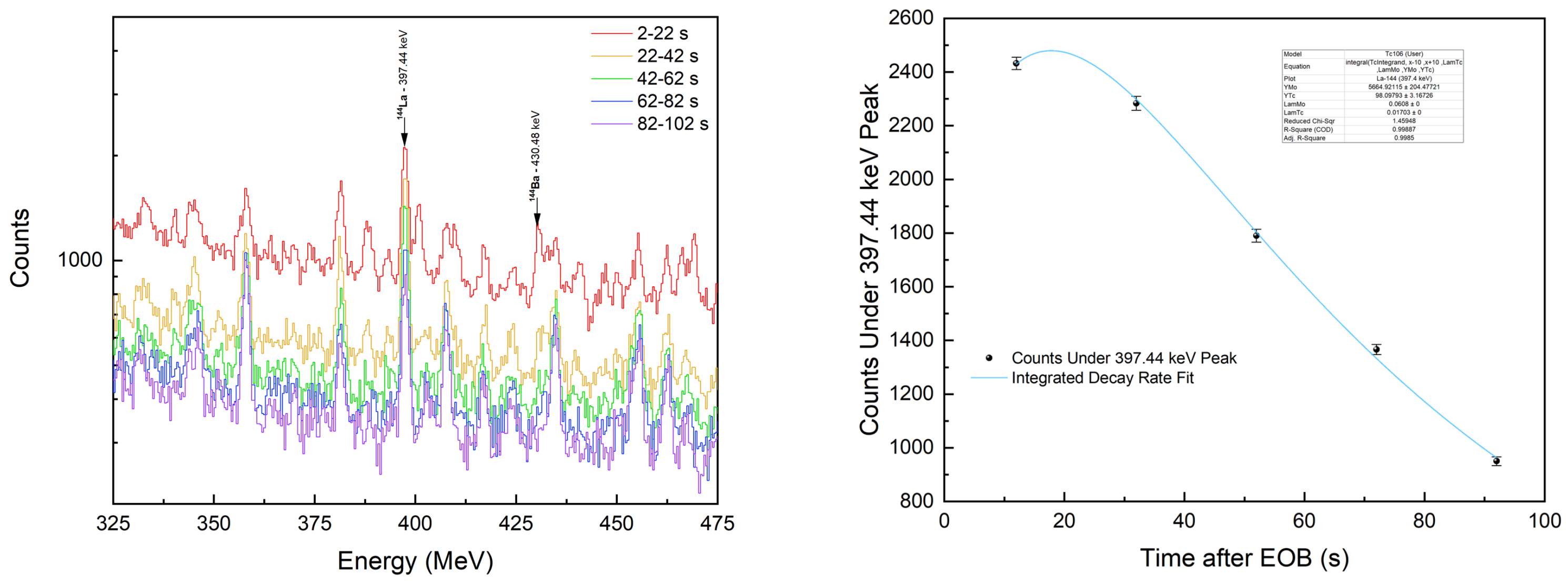

- Fit the individual bins with integrated activity to verify $\lambda$

- Also to solve for $\mathrm{N}_{0}$ for all contributing $\mathrm{R}(t)=\int_{x-10}^{x+10} \frac{\lambda_{L a 144} \lambda_{B a 144}}{\lambda_{L a 144}-\lambda_{B a 144}} N_{L a 144,0}\left[e^{-\lambda_{B a 144} x}-e^{-\lambda_{L a 144} x}\right]+\lambda_{B a 144} N_{B a 144,0} e^{-\lambda_{B a 144} x} d E$ nuclides 


\section{Photofissions per pulse from photonuclear cross sections}

- Need photofission event yield per pulse for absolute FPY calculations

- For ${ }^{238} \mathrm{U}$ photofission yield per pulse, the ratio of $\sigma(\gamma, f) / \sigma(\gamma, n)$ and ${ }^{237} \mathrm{U}$ production from $208.0 \mathrm{keV}$ $\mathrm{Y}$-ray peak

- For ${ }^{232}$ Th photofission yield per pulse, the ratio of $\sigma_{\mathrm{U} 238}(\mathrm{Y}, \mathrm{f}) / \sigma_{\mathrm{Th} 232}(\mathrm{Y}, \mathrm{f})$

Photofission Yield Per Linac Pulse

\begin{tabular}{cccc}
\hline \hline Target & $\mathrm{B}(\mathrm{MeV})$ & $\left\langle E^{*}(B)\right\rangle(\mathrm{MeV})$ & Fission Events Per Pulse \\
${ }^{238} \mathrm{U}$ & 8 & $6.78 \pm 0.01$ & $493 \pm 13$ \\
${ }^{238} \mathrm{U}$ & 14 & $10.30 \pm 0.01$ & $11,976 \pm 330$ \\
${ }^{238} \mathrm{U}$ & 20 & $12.59 \pm 0.01$ & $123,698 \pm 6,272$ \\
${ }^{232} \mathrm{Th}$ & 8 & $6.74 \pm 0.02$ & $374 \pm 10$ \\
${ }^{232} \mathrm{Th}$ & 14 & $9.22 \pm 0.01$ & $4951 \pm 156$ \\
${ }^{232} \mathrm{Th}$ & 20 & $11.50 \pm 0.03$ & $40,011 \pm 1552$
\end{tabular}

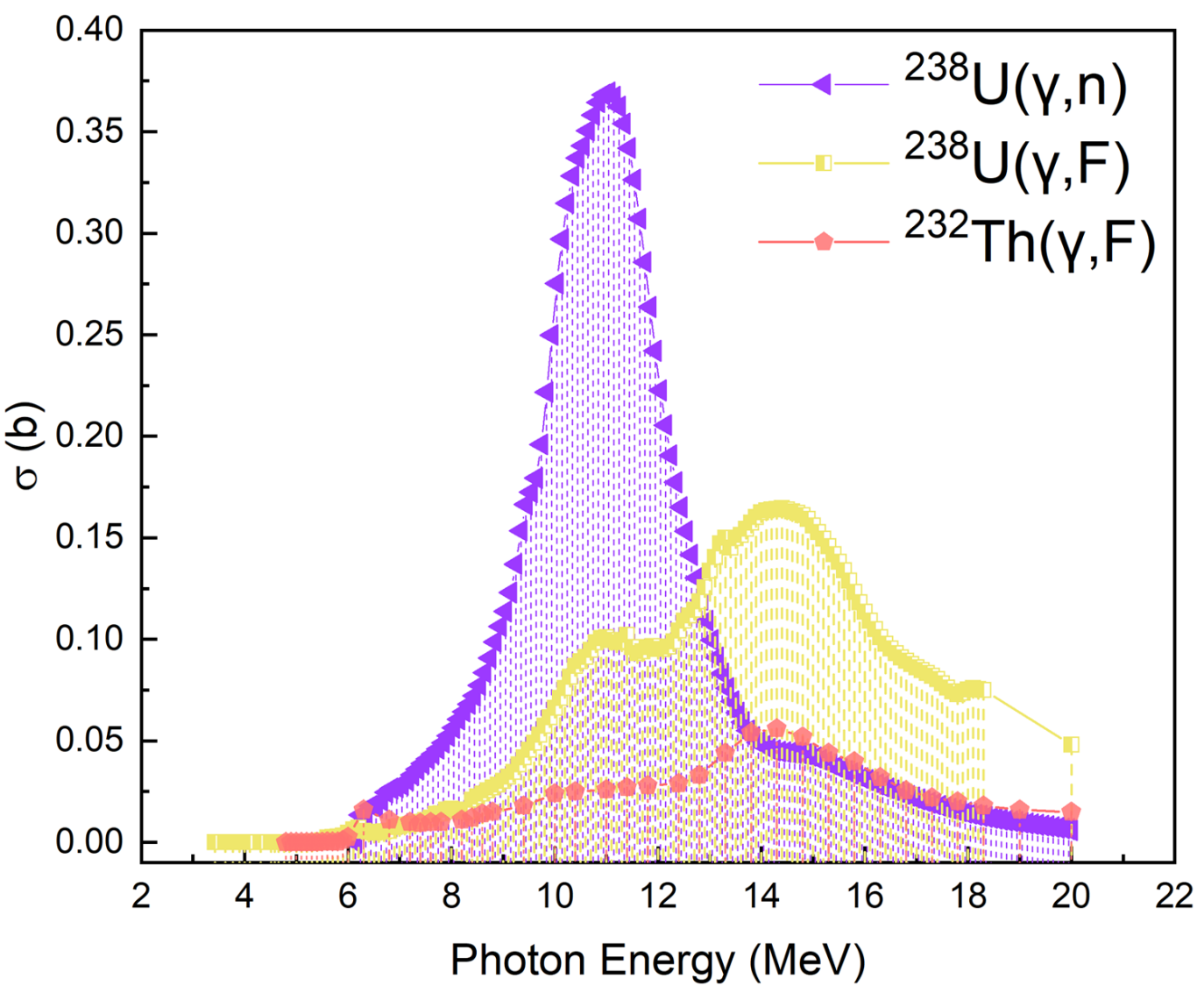




\section{Results}

IVI Idaho National Laboratory Oregon State 


\section{U Cumulative Fission Product Yields}

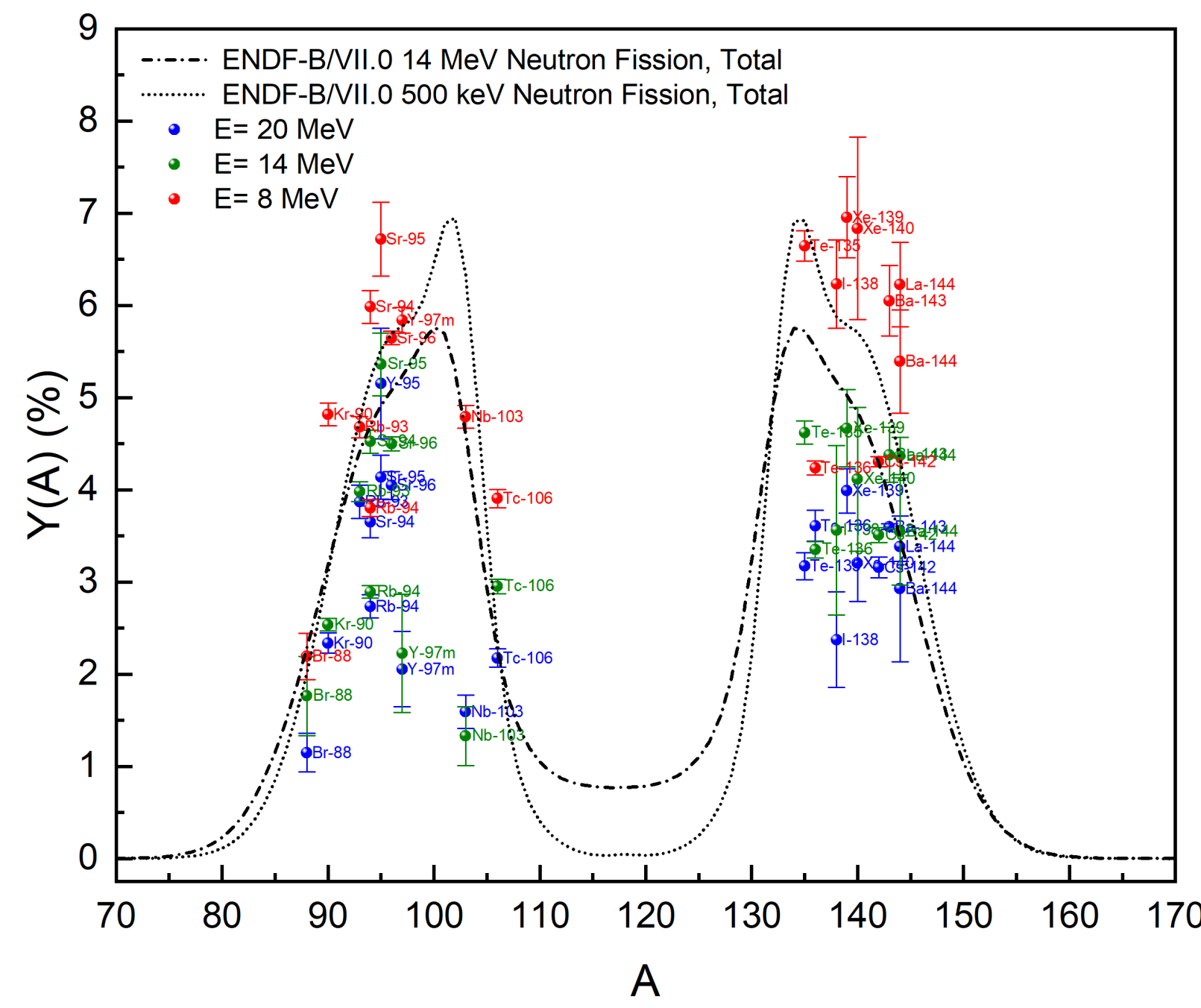

\begin{tabular}{|c|c|c|c|c|c|c|c|c|}
\hline \multicolumn{9}{|c|}{ Cumulative Photofission Product Yields for ${ }^{238} \mathrm{U}$} \\
\hline A & $z$ & Nuclide & $T_{1 / 2}(s)$ & $\beta$ & Energy (keV) & $\mathrm{B}=20 \mathrm{MeV}$ & $\mathrm{B}=14 \mathrm{MeV}$ & $\mathrm{B}=8 \mathrm{MeV}$ \\
\hline 88 & 35 & ${ }^{88} \mathrm{Br}$ & 16.34(8) & $0.625(3)$ & $775.28(6)$ & $0.01149(21)$ & $0.01762(43)$ & $0.02193(25)$ \\
\hline 90 & 36 & ${ }^{90} \mathrm{Kr}$ & $32.32(2)$ & $0.390(3)$ & $1118.69(5)$ & $0.02337(11)$ & $0.02536(7)$ & $0.04817(12)$ \\
\hline 93 & 37 & ${ }^{93} \mathrm{Rb}$ & $5.84(3)$ & $0.202(1)$ & 432.61(3) & $0.03870(18)$ & $0.03980(11)$ & $0.04679(11)$ \\
\hline 94 & 37 & ${ }^{94} \mathrm{Rb}$ & $2.702(5)$ & $0.610(4)$ & $836.9(1)$ & $0.02736(13)$ & $0.02893(7)$ & $0.03802(9)$ \\
\hline 94 & 38 & ${ }^{94} \mathrm{Sr}$ & $75.3(2)$ & $0.942(9)$ & $1427.7(1)$ & $0.03652(17)$ & $0.04522(12)$ & $0.05983(18)$ \\
\hline 95 & 38 & ${ }^{95} \mathrm{Sr}$ & 23.9(14) & $0.226(12)$ & $685.6(-)$ & $0.04136(24)$ & $0.05361(31)$ & $0.06717(40)$ \\
\hline 96 & 38 & ${ }^{96} \mathrm{Sr}$ & $1.07(1)$ & $0.719(3)$ & $809.4(3)$ & $0.04045(15)$ & $0.04499(8)$ & $0.05647(73)$ \\
\hline 97 & 39 & $97 \mathrm{mY}$ & $1.17(3)$ & $0.921(2)$ & $1103.3(3)$ & $0.02054(41)$ & $0.02226(64)$ & $0.05840(14)$ \\
\hline 103 & 41 & ${ }^{103} \mathrm{Nb}$ & $1.5(2)$ & $1.00(6)$ & $102.561(3)$ & $0.01594(7)$ & $0.01329(3)$ & $0.04790(3)$ \\
\hline 106 & 43 & ${ }^{106} \mathrm{Tc}$ & $35.6(6)$ & $0.558(17)$ & $270.096(9)$ & $0.02175(10)$ & $0.02951(8)$ & $0.03904(10)$ \\
\hline 135 & 52 & ${ }^{135} \mathrm{Te}$ & 19(2) & $0.279(12)$ & $603.70(3)$ & $0.03172(15)$ & $0.04621(13)$ & $0.06645(17)$ \\
\hline 136 & 52 & ${ }^{136} \mathrm{Te}$ & $17.63(9)$ & $0.182(2)$ & 578.75(3) & $0.03610(17)$ & $0.03350(9)$ & $0.04236(7)$ \\
\hline 138 & 53 & $138 \mid$ & $6.26(3)$ & $0.56(3)$ & $588.825(18)$ & $0.02374(52)$ & $0.03561(92)$ & $0.06232(48)$ \\
\hline 139 & 54 & ${ }^{139} \mathrm{Xe}$ & $39.68(14)$ & $0.217(6)$ & $296.53(7)$ & $0.03989(24)$ & $0.04667(42)$ & $0.06956(44)$ \\
\hline 140 & 54 & ${ }^{140} \mathrm{Xe}$ & $13.6(10)$ & $0.20(2)$ & $805.52(10)$ & $0.03206(42)$ & $0.04115(78)$ & $0.06835(99)$ \\
\hline 142 & 55 & ${ }^{142} \mathrm{Cs}$ & $1.684(14)$ & $0.128(3)$ & $1326.46(7)$ & $0.03157(11)$ & $0.03509(8)$ & $0.04304(5)$ \\
\hline 143 & 56 & ${ }^{143} \mathrm{Ba}$ & $14.5(3)$ & $0.156(13)$ & 798.79(2) & $0.03599(30)$ & $0.04390(30)$ & $0.05550(123)$ \\
\hline 144 & 56 & ${ }^{144} \mathrm{Ba}$ & $11.5(2)$ & $0.183(4)$ & $430.48(12)$ & $0.02925(79)$ & $0.03547(58)$ & $0.04304(5)$ \\
\hline 144 & 57 & ${ }^{144} \mathrm{La}$ & $40.8(4)$ & $0.943(2)$ & 397.44(9) & $0.03384(16)$ & $0.04368(20)$ & $0.06226(36)$ \\
\hline
\end{tabular}

PVL Idaho National laboratory

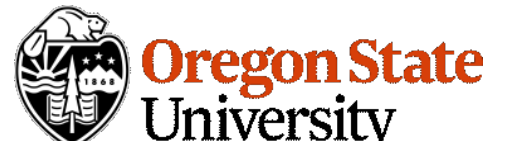

$$
\begin{aligned}
& \left\langle E^{*}(B)\right\rangle=12.59 \pm 0.01 \mathrm{MeV} \\
& \left\langle E^{*}(B)\right\rangle=10.30 \pm 0.01 \mathrm{MeV} \\
& \left\langle E^{*}(B)\right\rangle=6.78 \pm 0.01 \mathrm{MeV}
\end{aligned}
$$




\section{Th Cumulative Fission Product Yields}

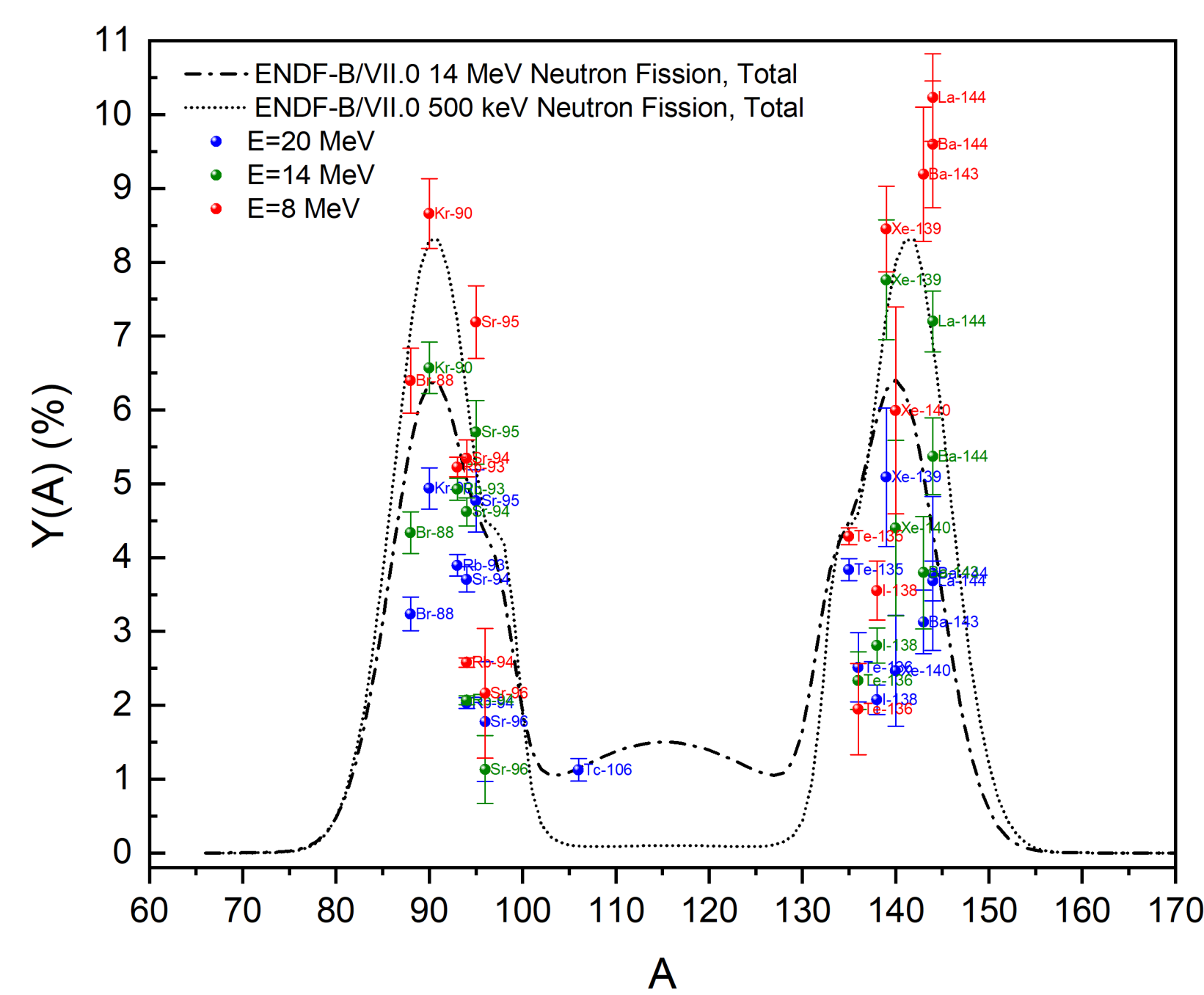

\begin{tabular}{|c|c|c|c|c|c|c|c|c|}
\hline \multicolumn{9}{|c|}{ Cumulative Photofission Product Yields for ${ }^{232} \mathrm{Th}$} \\
\hline A & z & Nuclide & $T_{1 / 2}(s)$ & $\beta$ & Energy (keV) & $B=20 \mathrm{MeV}$ & $\mathrm{B}=14 \mathrm{MeV}$ & $\mathrm{B}=8 \mathrm{MeV}$ \\
\hline 88 & 35 & ${ }^{88} \mathrm{Br}$ & $16.34(8)$ & $0.625(3)$ & $775.28(6)$ & $0.03236(23)$ & $0.04337(28)$ & $0.06395(44)$ \\
\hline 90 & 36 & ${ }^{90} \mathrm{Kr}$ & $32.32(2)$ & $0.390(3)$ & $1118.69(5)$ & $0.04936(28)$ & $0.06570(35)$ & $0.08660(47)$ \\
\hline 93 & 37 & ${ }^{93} \mathrm{Rb}$ & $5.84(3)$ & $0.202(1)$ & 432.61(3) & $0.03894(15)$ & $0.04925(15)$ & $0.05225(13)$ \\
\hline 94 & 37 & ${ }^{94} \mathrm{Rb}$ & $2.702(5)$ & $0.610(4)$ & $836.9(1)$ & $0.02028(7)$ & $0.02067(6)$ & $0.02579(9)$ \\
\hline 94 & 38 & ${ }^{94} \mathrm{Sr}$ & $75.3(2)$ & $0.942(9)$ & $1427.7(1)$ & $0.03705(17)$ & $0.04622(19)$ & $0.05344(25)$ \\
\hline 95 & 38 & ${ }^{95} \mathrm{Sr}$ & 23.9(14) & $0.226(12)$ & $685.6(-)$ & $0.04683(42)$ & $0.05698(43)$ & $0.07190(49)$ \\
\hline 96 & 38 & $96 \mathrm{Sr}$ & $1.07(1)$ & $0.719(3)$ & $809.4(3)$ & $0.01778(81)$ & $0.01128(46)$ & $0.02163(73)$ \\
\hline 106 & 43 & ${ }^{106} \mathrm{Tc}$ & $35.6(6)$ & $0.558(17)$ & $270.096(9)$ & $0.01126(15)$ & - & - \\
\hline 135 & 52 & ${ }^{135} \mathrm{Te}$ & 19(2) & $0.279(12)$ & $603.70(3)$ & $0.03836(15)$ & - & $0.04288(11)$ \\
\hline 136 & 52 & ${ }^{136} \mathrm{Te}$ & $17.63(9)$ & $0.182(2)$ & $578.75(3)$ & $0.02513(75)$ & $0.02332(39)$ & $0.01948(62)$ \\
\hline 138 & 53 & $138 \mid$ & $6.26(3)$ & $0.56(3)$ & $588.825(18)$ & $0.02074(20)$ & $0.02810(24)$ & $0.03552(40)$ \\
\hline 139 & 54 & ${ }^{139} \mathrm{Xe}$ & $39.68(14)$ & $0.217(6)$ & $296.53(7)$ & $0.05088(94)$ & $0.07762(81)$ & $0.08452(58)$ \\
\hline 140 & 54 & ${ }^{140} \mathrm{Xe}$ & $13.6(10)$ & $0.20(2)$ & $805.52(10)$ & $0.02467(75)$ & $0.04399(119)$ & $0.05993(140)$ \\
\hline 143 & 56 & ${ }^{143} \mathrm{Ba}$ & $14.5(3)$ & $0.156(13)$ & 798.79(2) & $0.03054(43)$ & $0.03704(76)$ & $0.08972(91)$ \\
\hline 144 & 56 & ${ }^{144} \mathrm{Ba}$ & $11.5(2)$ & $0.183(4)$ & $430.48(12)$ & $0.03785(104)$ & $0.05370(52)$ & $0.09596(86)$ \\
\hline 144 & 57 & ${ }^{144} \mathrm{La}$ & $40.8(4)$ & $0.943(2)$ & $397.44(9)$ & $0.03682(27)$ & $0.07199(41)$ & $0.10232(59)$ \\
\hline
\end{tabular}$$
\left\langle E^{*}(B)\right\rangle=11.50 \pm 0.03 \mathrm{MeV}
$$$$
\left\langle E^{*}(B)\right\rangle=9.22 \pm 0.01 \mathrm{MeV}
$$$$
\left\langle E^{*}(B)\right\rangle=6.74 \pm 0.02 \mathrm{MeV}
$$ 


\section{Distribution of ${ }^{238} \mathrm{U}$ and ${ }^{232} \mathrm{Th}$ FPY}

\section{Uranium-238}

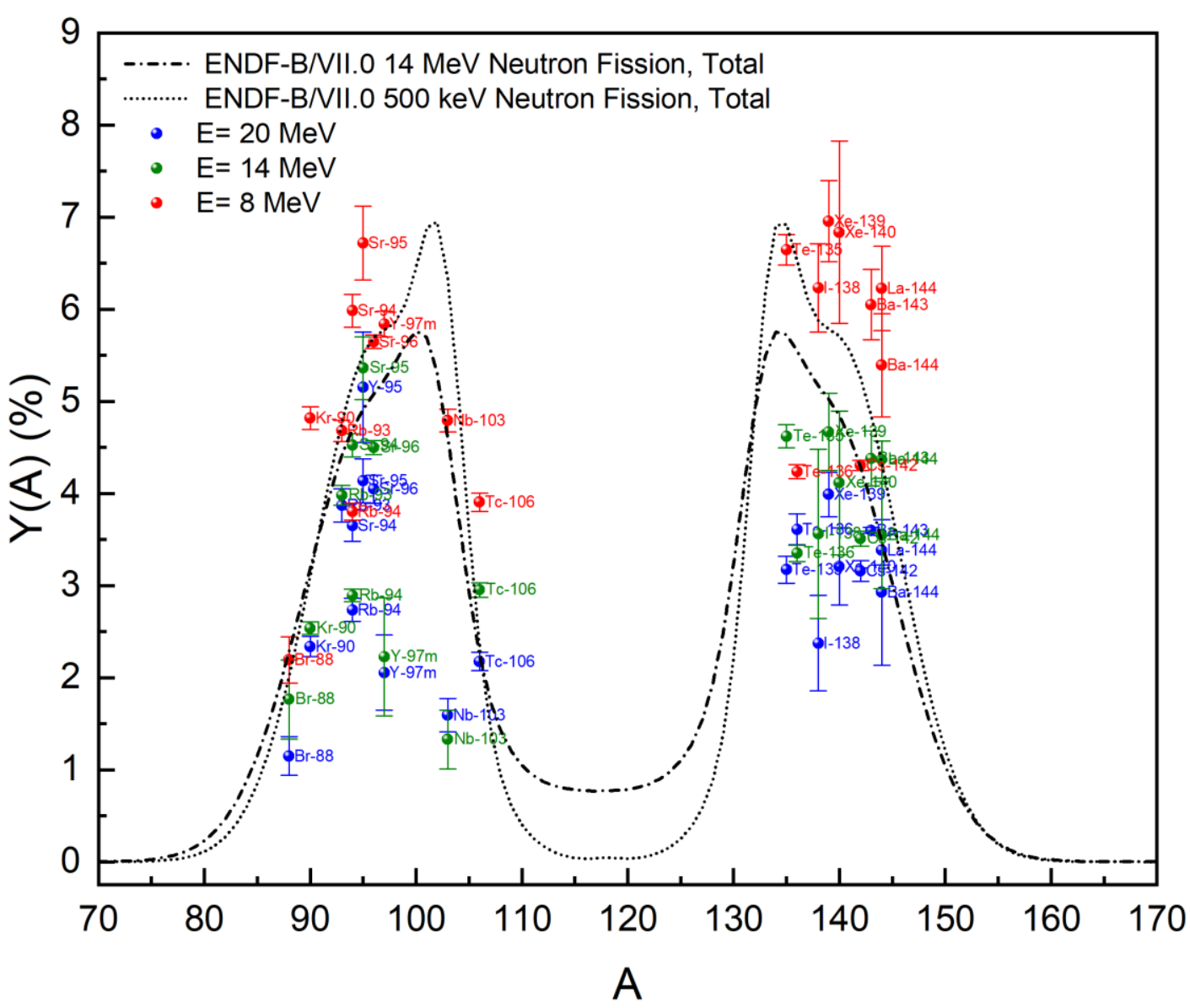

Thorium-232

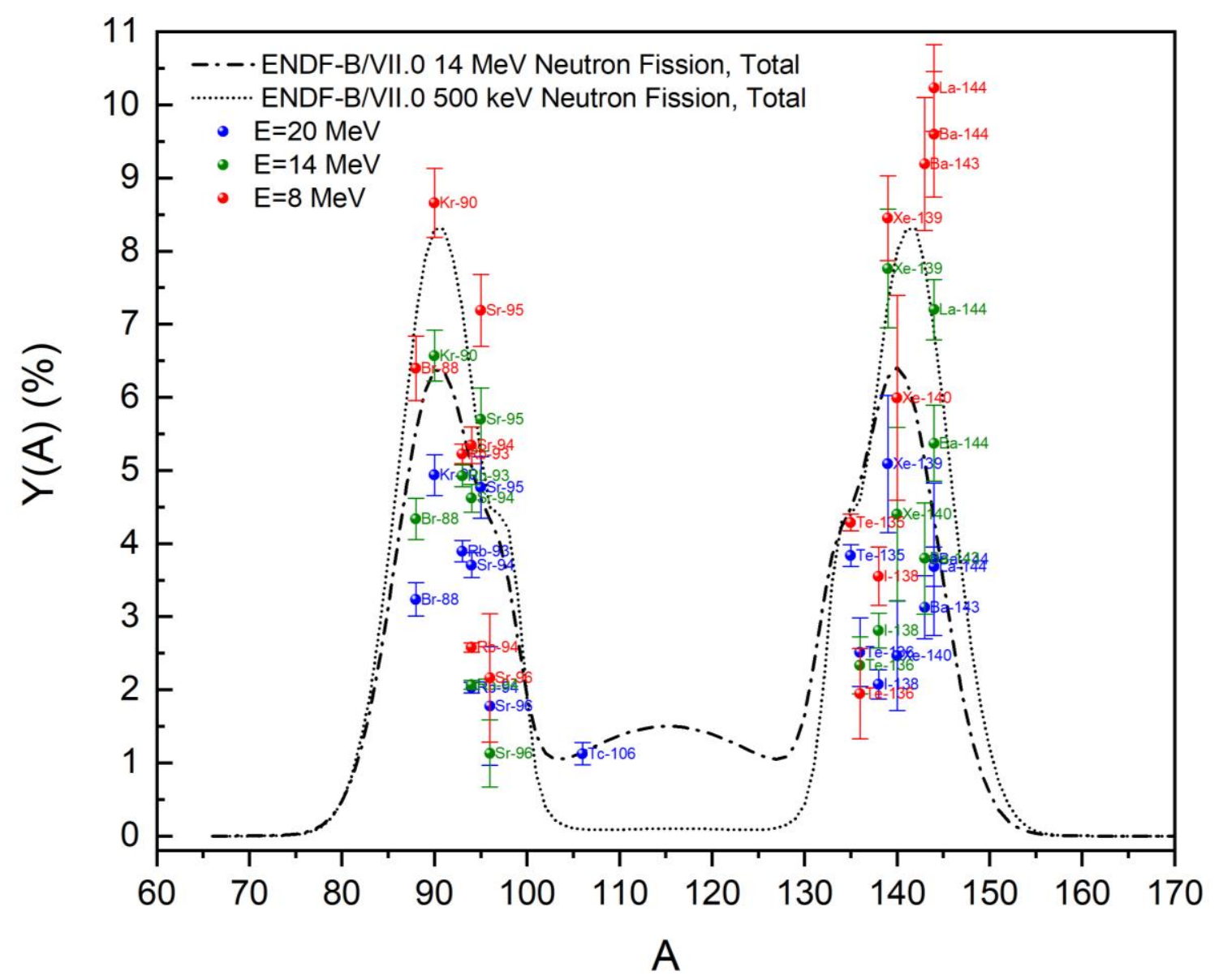




\section{Conclusion}

IT Idaho National Laboratory Oregon State 


\section{Conclusions}

- Short-lived products reported for ${ }^{238} \mathrm{U}$ and ${ }^{232} \mathrm{Th}$ for 3 endpoint energies, reported with both endpoint and excitation energies to allow for comparison

- CFPYs for $20 \mathrm{MeV}$ good agreement with DT neutron fission

- CFPYs for 14 and $8 \mathrm{MeV}$ reflect expected distribution using the ENDF-B/VII.0 fractional mass yields and multimode -fission model

- Absolute uncertainty reported with all factors considered where data was available or acquired through simulation

- Photofission product yields measured for lower ( $\leq 12 \mathrm{MeV})$ excitation energy to be most useful for forensics applications 


\section{Questions?}

I1 Idaho National Laboratory OregonState

ANS 29 


\section{Supplemental Slides}




\section{HPGe Detector and Instrumentation}

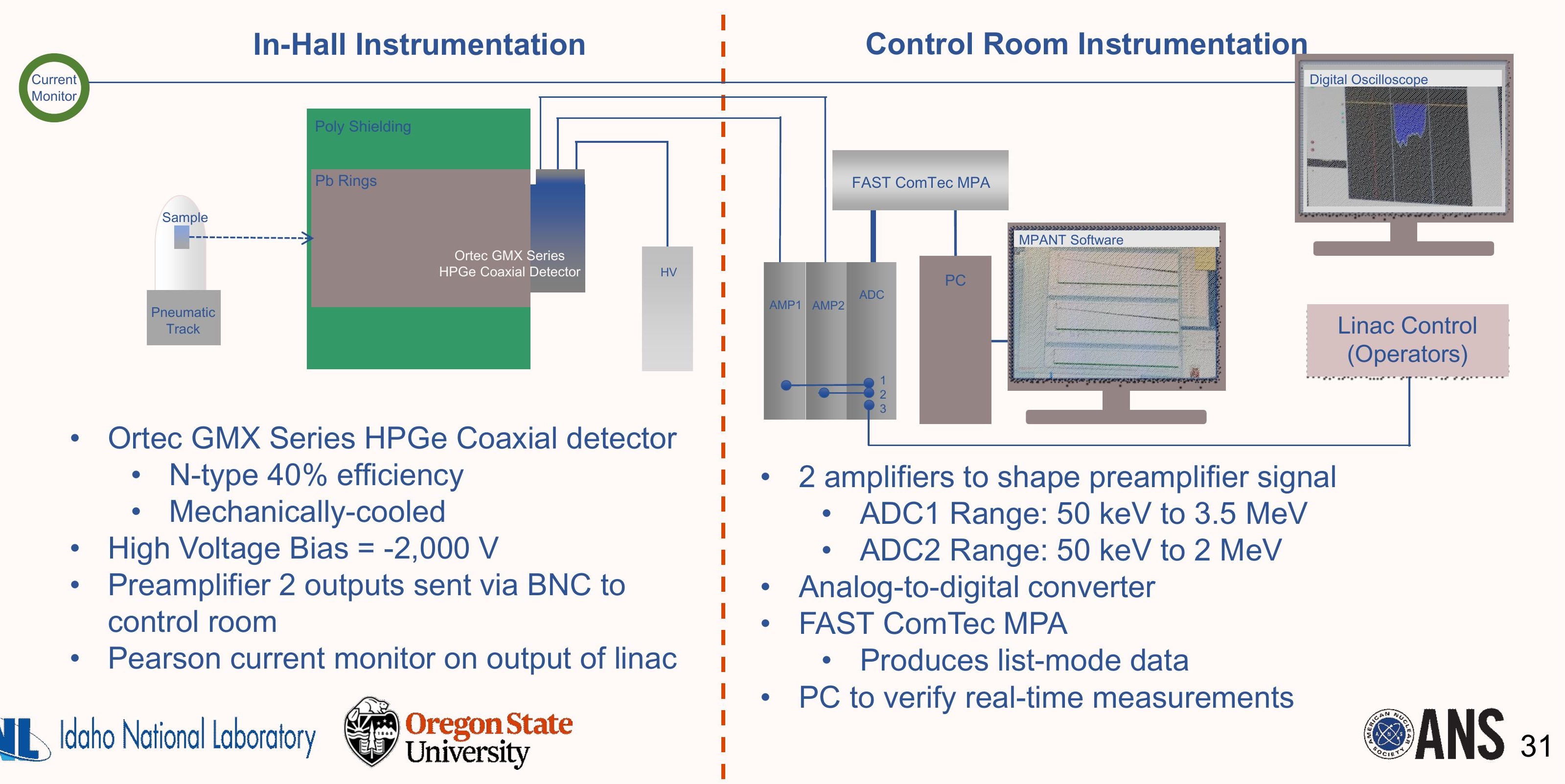




\section{Bremsstrahlung X-Ray Production}

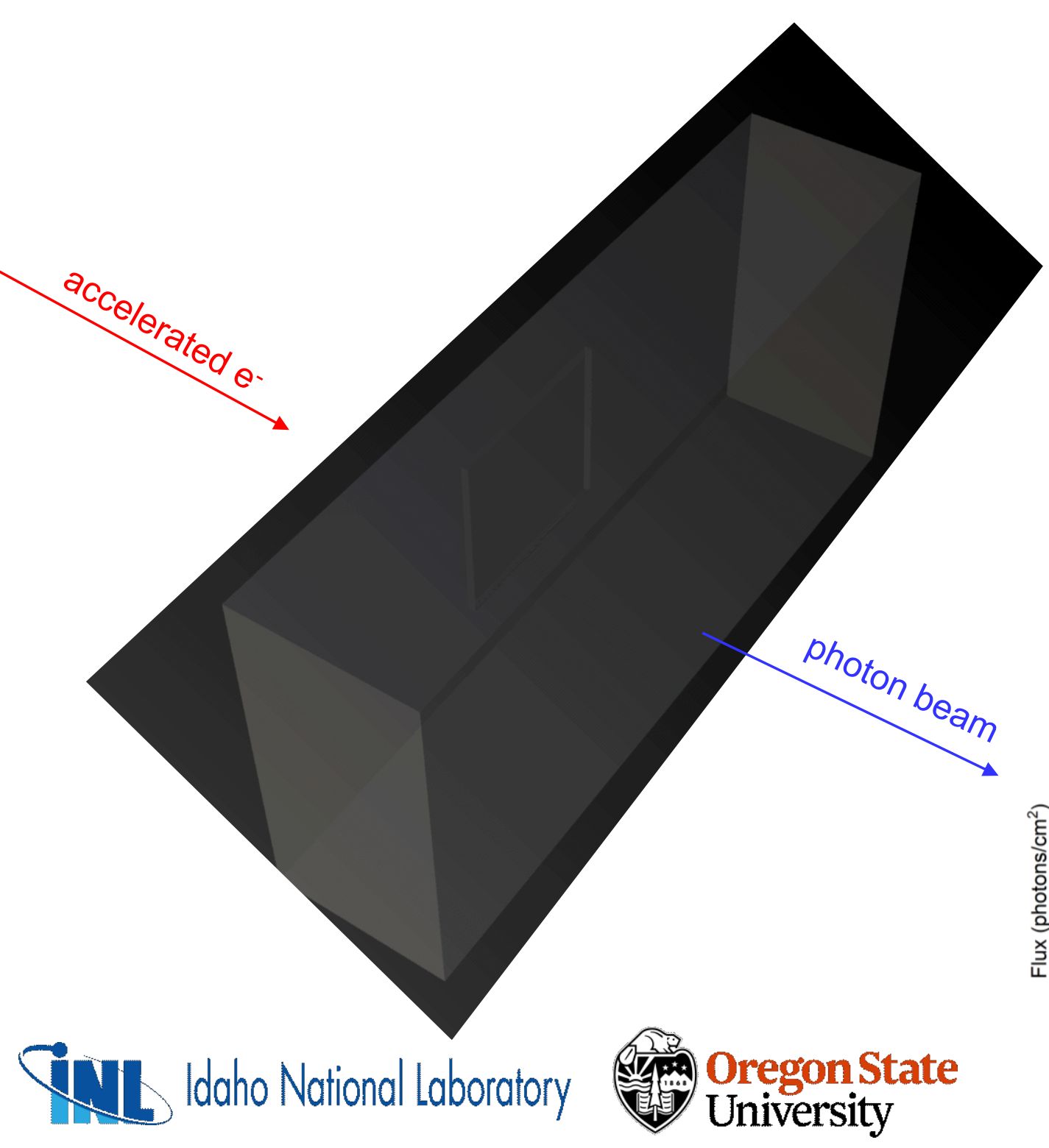

- Linac produces high energy electron beam

- Operators control energy of these accelerated $\mathrm{e}^{-}$

- $0.22 \mathrm{~cm}$ thick W radiator/converter to convert electron beam to bremsstrahlung photon spectra

-W Converter is embedded in aluminum beam scrub, requires intense cooling

- Bremsstrahlung X-ray spectra produced from deceleration of $e^{-}$beam

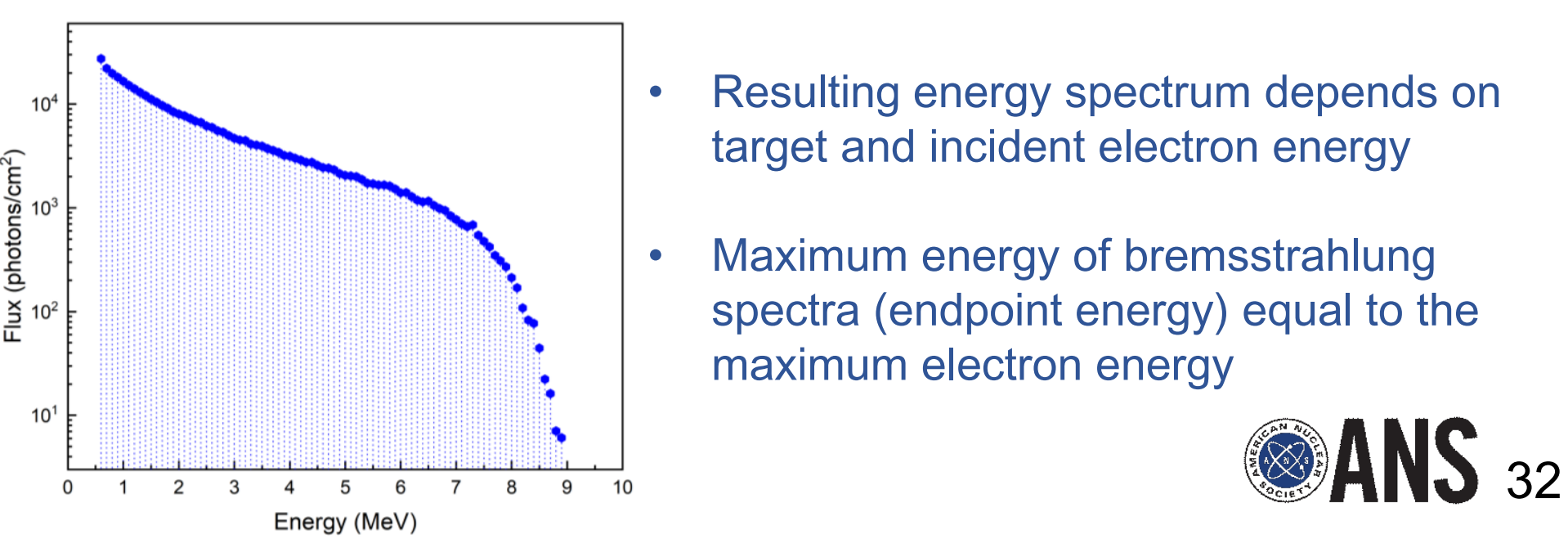




\section{Integral Decay Rate Fit}
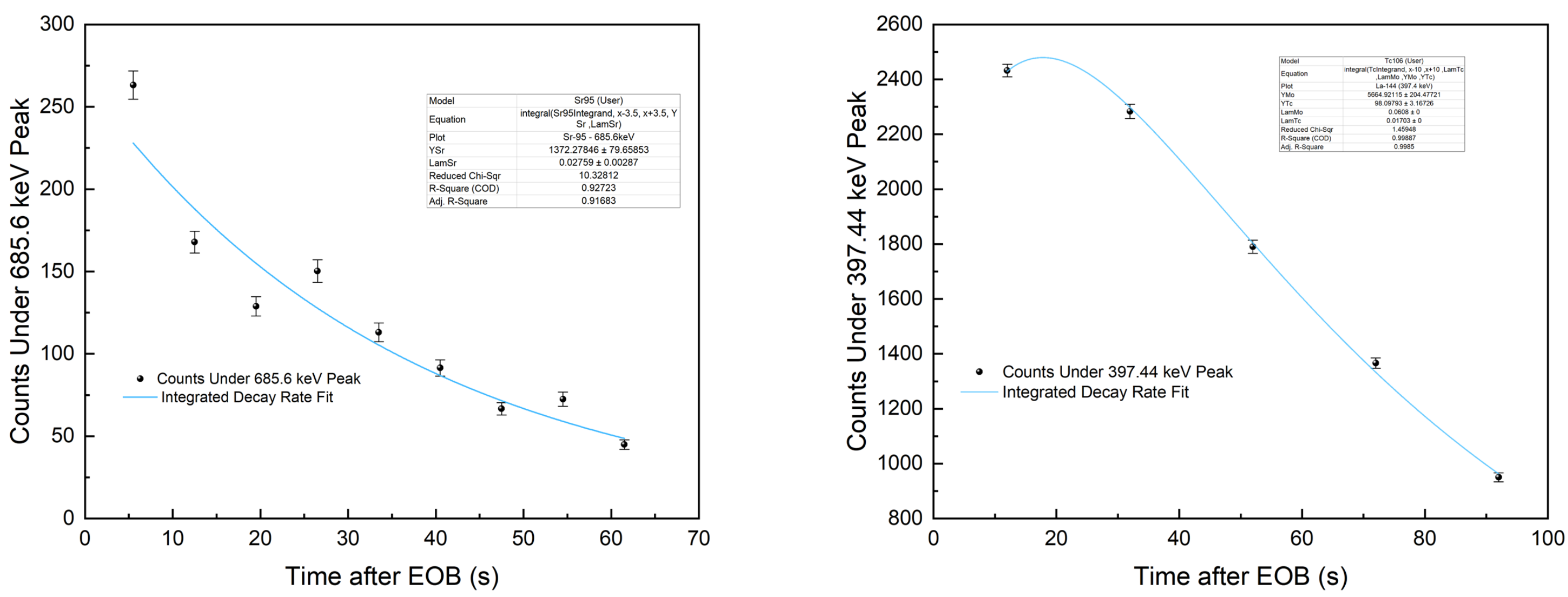

$$
\mathrm{R}(t)=\int_{x-2.5}^{x+2.5} \lambda_{S r 95} N_{S r 95,0} e^{-\lambda_{S r 95} x} d E
$$

- If parent decays fully prior to counts, not contributing to peak and not included

$$
{ }^{95} \mathrm{Kr}\left(\mathrm{T}_{1 / 2}=114 \mathrm{~ms}\right) \rightarrow{ }^{95} \mathrm{Rb}\left(\mathrm{T}_{1 / 2}=377 \mathrm{~ms}\right) \rightarrow{ }^{95} \mathrm{Sr}\left(\mathrm{T}_{1 / 2}=25.1 \mathrm{~s}\right)
$$




\section{Optimization of parsing}

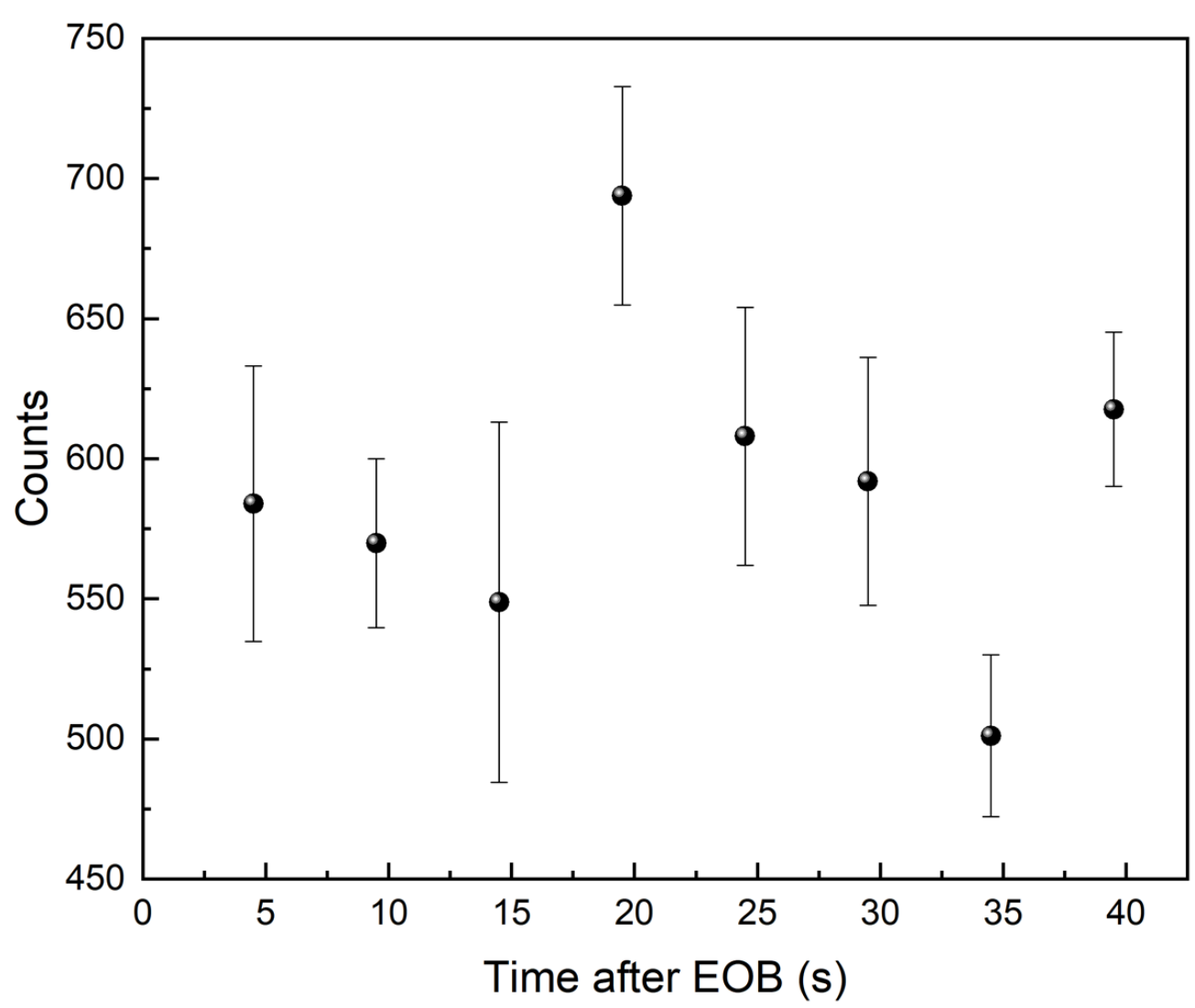

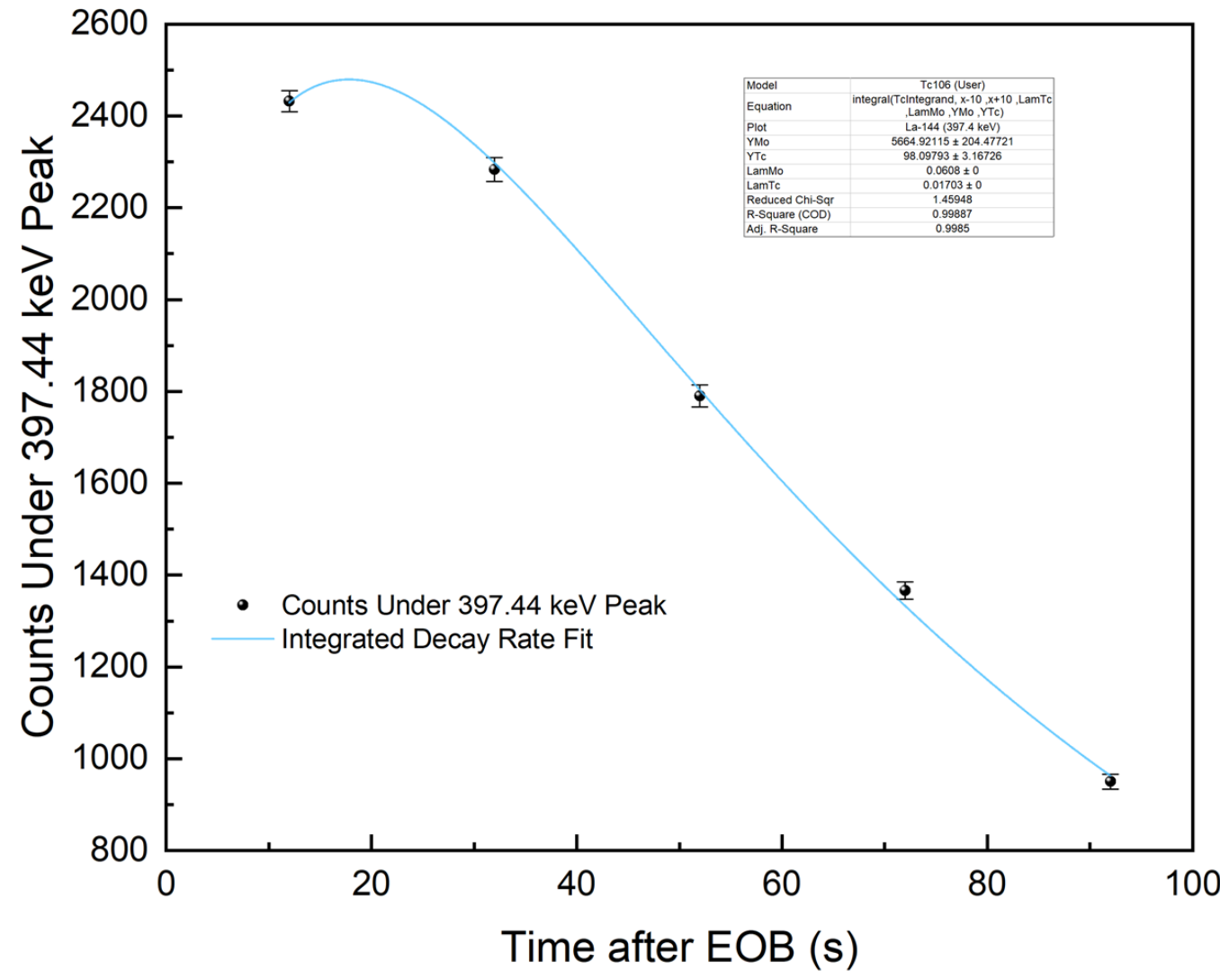

- Parsed to timing bins about $1 / 2$ the half-life of nuclide of interest to optimize statistics

- Some parsing resulted in sufficient statistics to fit for solving 


\section{HPGe Detector Efficiency}

$\mathrm{ADC} 2$

- Model of absolute efficiency for the in-hall HPGe detection setup to include uncertainty at any energy: ADC1 and ADC2

- Includes uncertainty contribution from:

- Nuclear data accessed from $\operatorname{ENSDF}^{2}\left(T_{1 / 2}\right.$ and $\beta$ )

- Activity reported for each calibrated check source from the manufacturer

- Counts measured under y-ray peak

- Use of partial error and micro-correlation matrix to include dependence from $y$-ray energies originating from the same nuclide

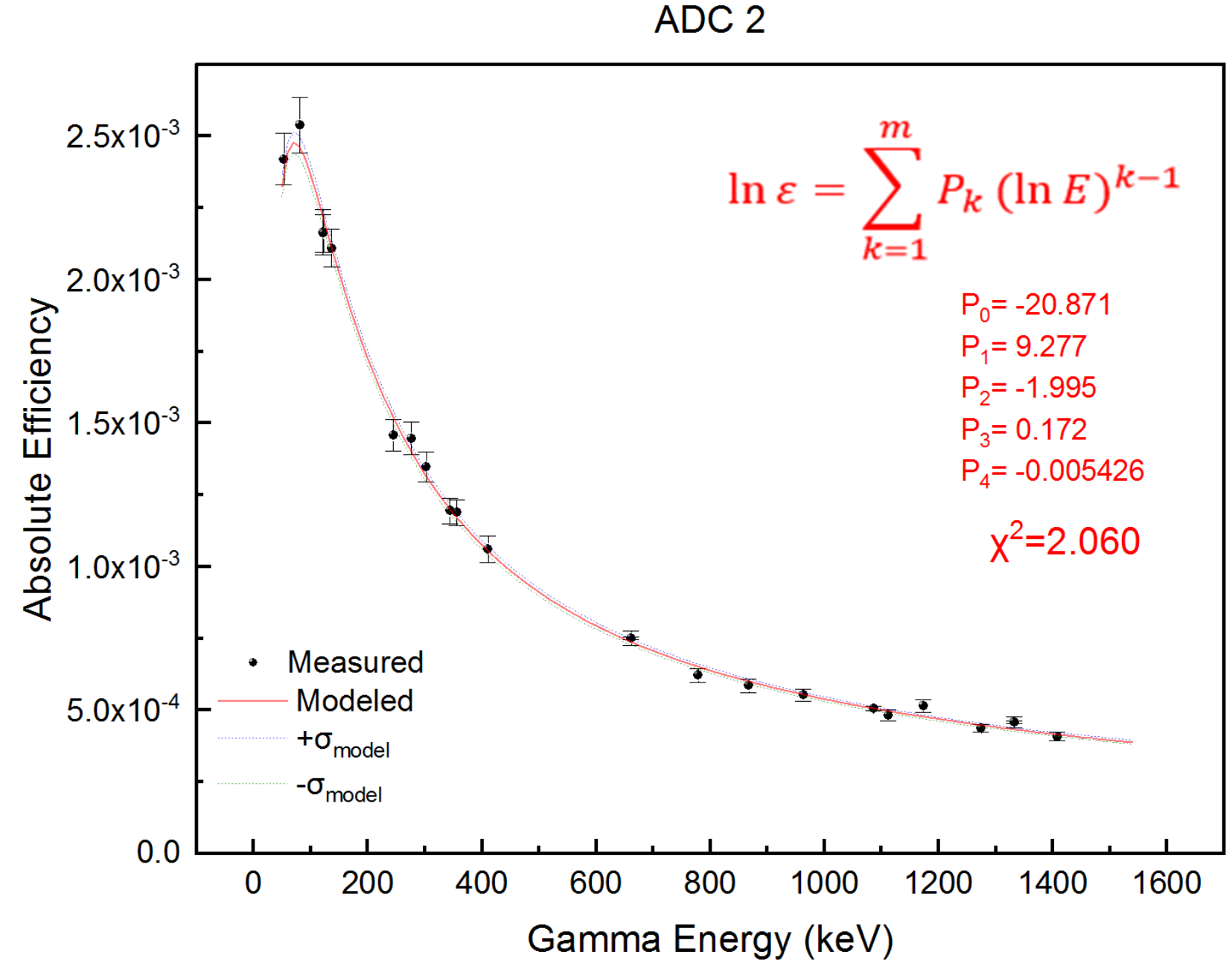

${ }^{2}$ ENSDF: Evaluated Nuclear Structure Data File Search and Retrieval, National Nuclear Data Center. https://www.nndc.bnl.gov/ensdf/

L. Geraldo and D. Smith, "Covariance analysis and fitting of germanium gamma-ray detector efficiency calibration data," NIM A: vol. 290, no. 2, pp. 499-508, 1990. 


\section{Model of HPGe Response}
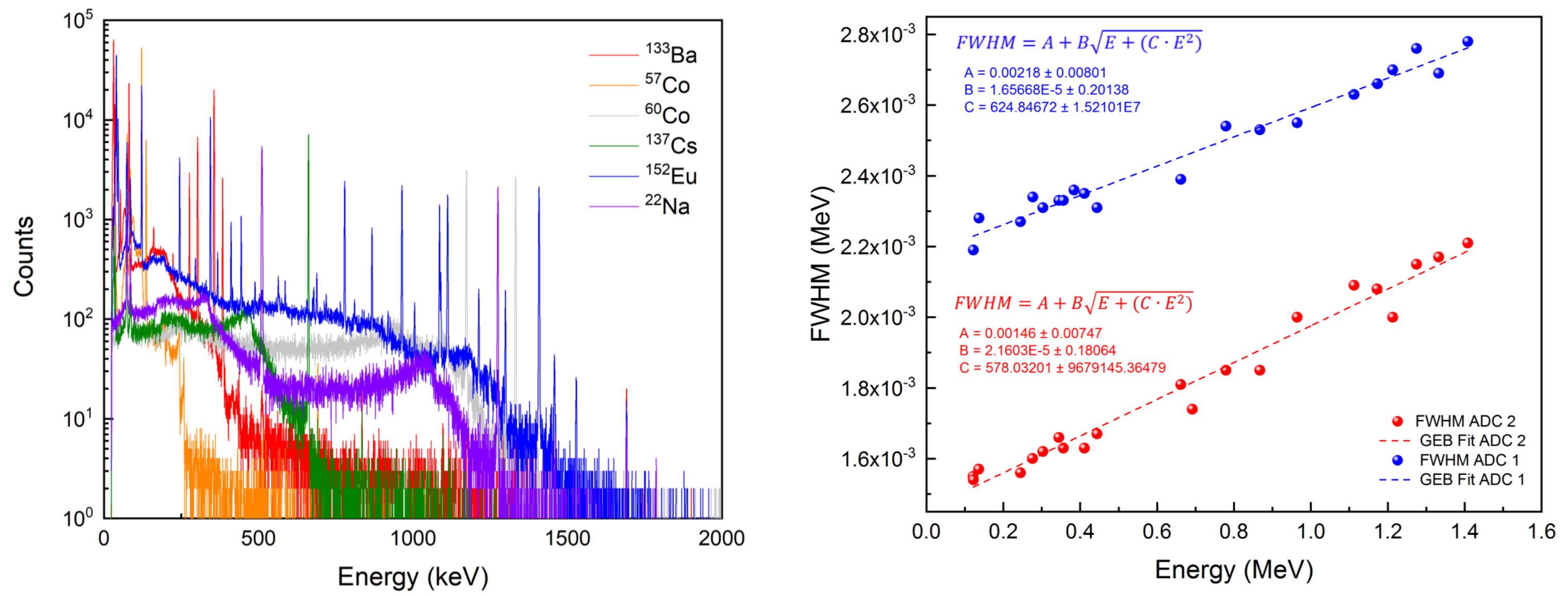

- High-fidelity model of high-purity germanium (HPGe) spectroscopic response using MCNP6

- Resolution of check source $y$-rays measured from energy calibration measurements and fit to determine FWHM 


\section{Model of HPGe Response}
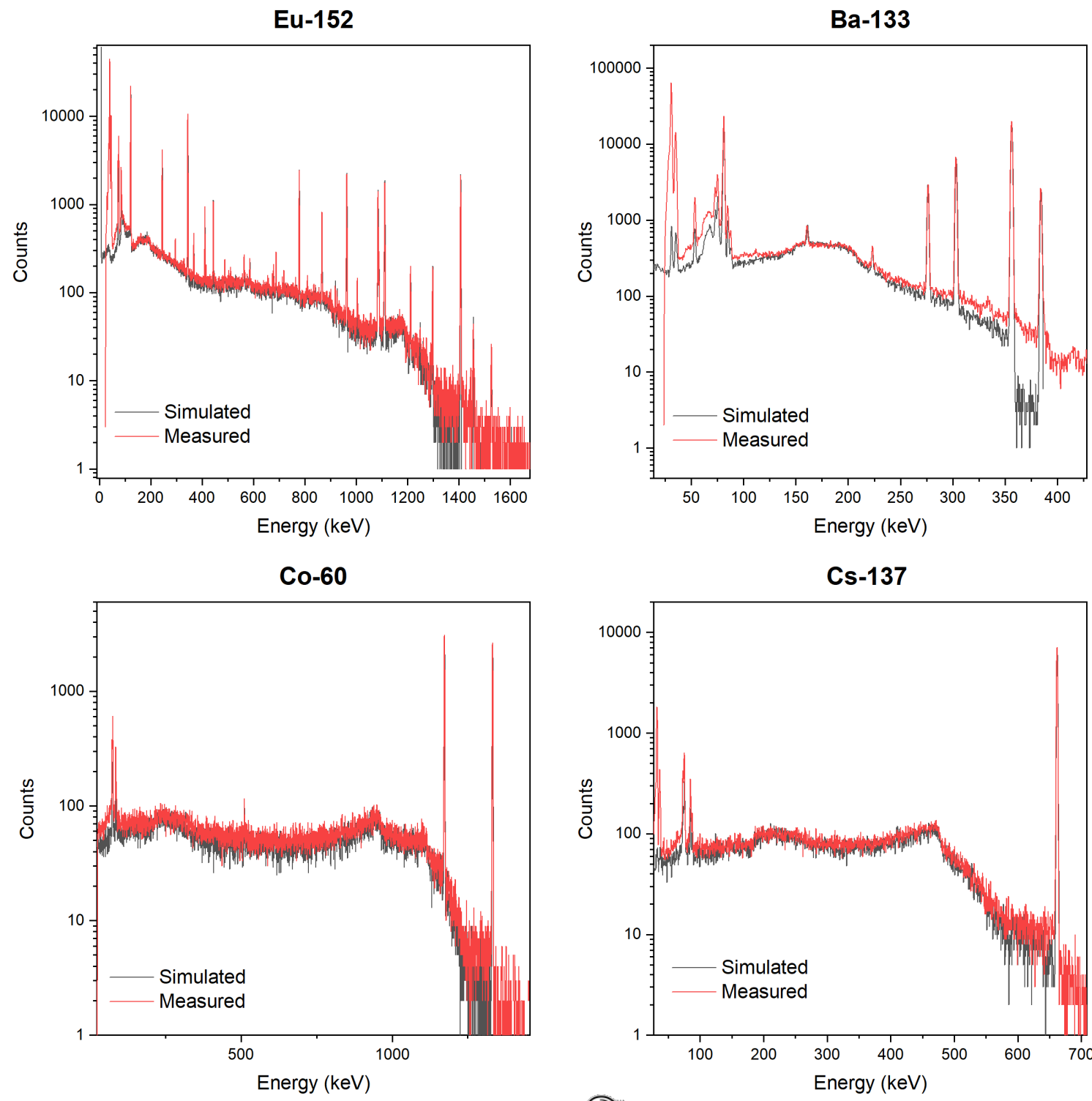

TII Idaho National Laboratory

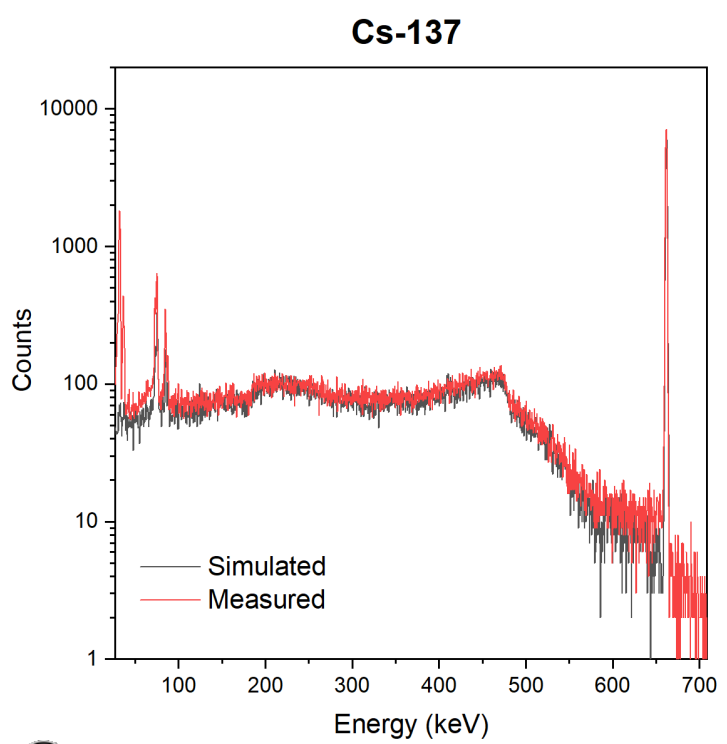

Oregon State E) University

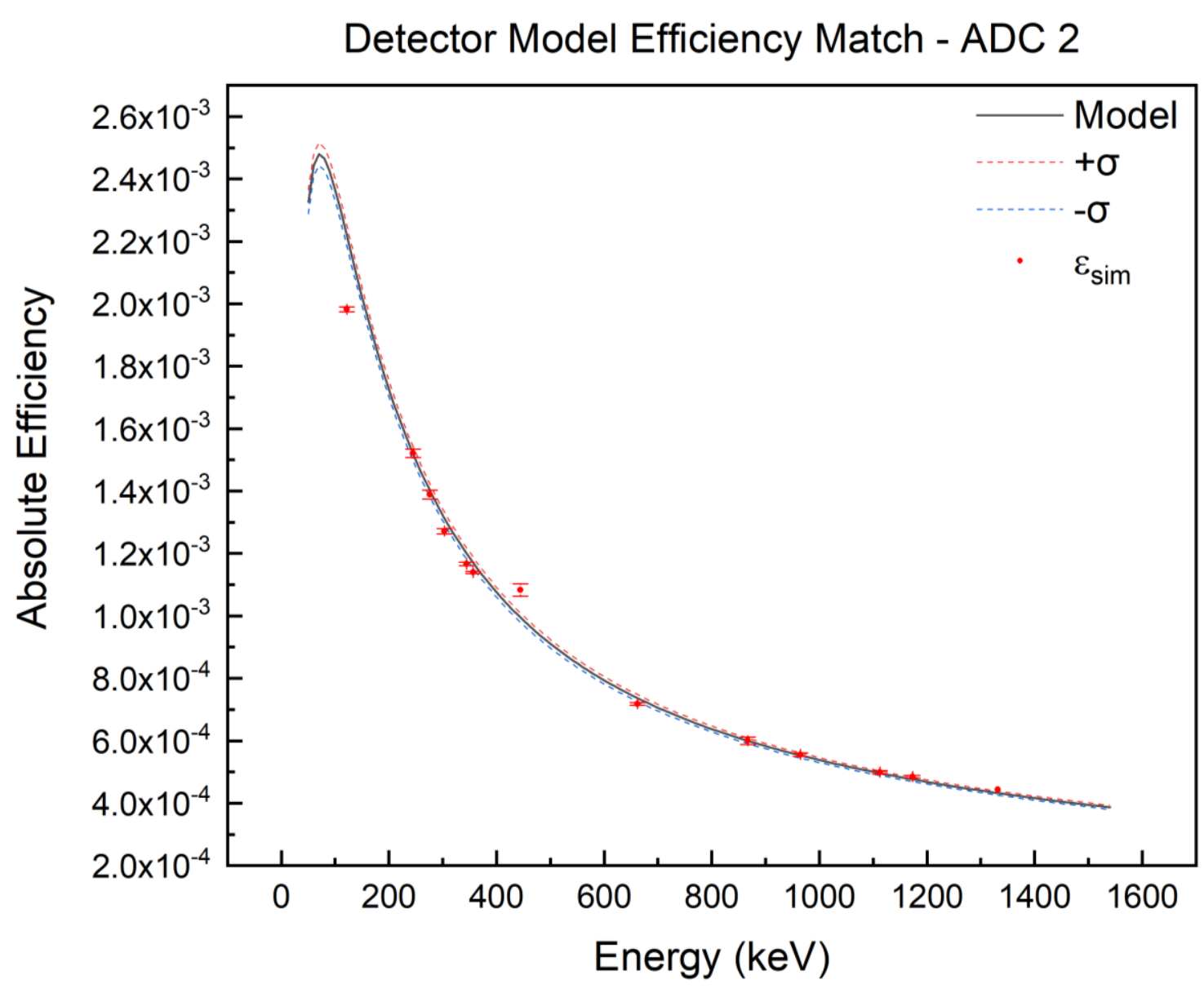

- Matched energy efficiency of model within 5\%

- Foil vs. Point Source verification 


\section{Photofissions per pulse from photonuclear cross sections}

- Need photofission event yield per pulse for absolute FPY calculations

- For ${ }^{238} \mathrm{U}$ photofission yield per pulse, the ratio of $\sigma(\gamma, \mathrm{f}) / \sigma(\mathrm{\gamma}, \mathrm{n})$ and ${ }^{237} \mathrm{U}$ production from $208.0 \mathrm{keV}$ $\mathrm{Y}$-ray peak

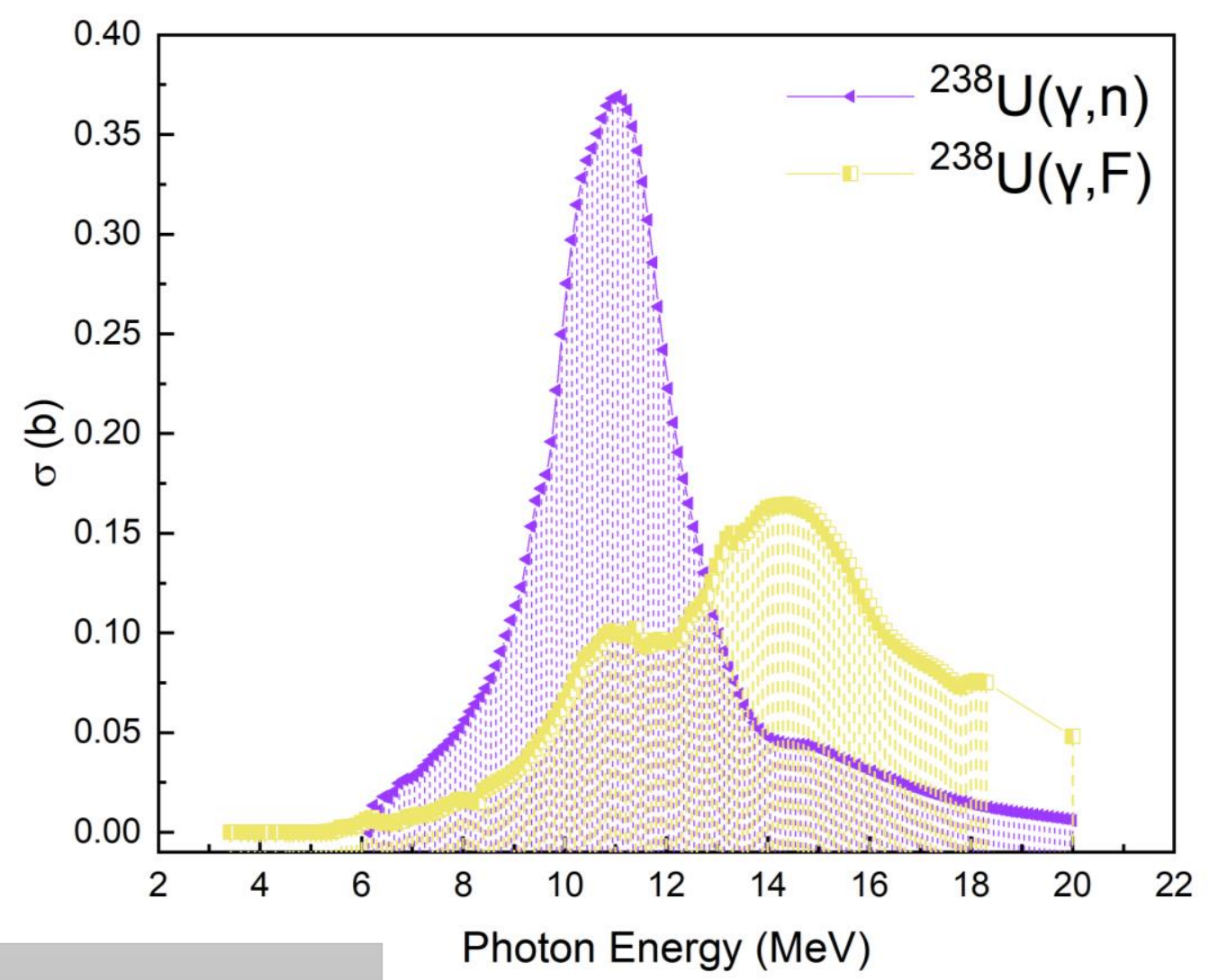

$Y_{U 238, f, p p}=\frac{\frac{C_{208}}{\beta \varepsilon A_{p}} \cdot \frac{R T}{L T}}{\sum_{M=0}^{\text {cycles }} \sum_{m=0}^{\text {pulses }} \int_{t_{1}}^{t_{2}} \sum_{i}^{M} e^{\left(-\lambda\left(T_{M}-T_{i}\right)+t+(m \tau)\right.} \lambda d t} \cdot \frac{\int_{0}^{B} \frac{\phi(B, E)}{d E} \sigma_{U 238, \gamma, f}(E) d E}{\int_{0}^{B} \frac{\phi(B, E)}{d E} \sigma_{U 238 \gamma, n}(E) d E}$

${ }^{1}$ ENDF-B/VII.0: Accessed from Janis 4.0, Nuclear Energy Agency, Organization for Economic Co-operation and Development. https://www.oecd-nea.org/janis/ 


\section{Photofissions per pulse from photonuclear cross sections}

- Need photofission event yield per pulse for absolute FPY calculations

- For ${ }^{232}$ Th photofission yield per pulse, the ratio of $\sigma_{\mathrm{U} 238}(\mathrm{Y}, \mathrm{f}) / \sigma_{\mathrm{Th} 232}(\mathrm{Y}, \mathrm{f})$

$$
Y_{T h 232, f, p p}=Y_{U 238, f, p p} \cdot \frac{\int_{0}^{B} \frac{\phi(B, E)}{d E} \sigma_{T h 232, \gamma, f}(E) d E}{\int_{0}^{B} \frac{\phi(B, E)}{d E} \sigma_{U 238, \gamma, f}(E) d E}
$$

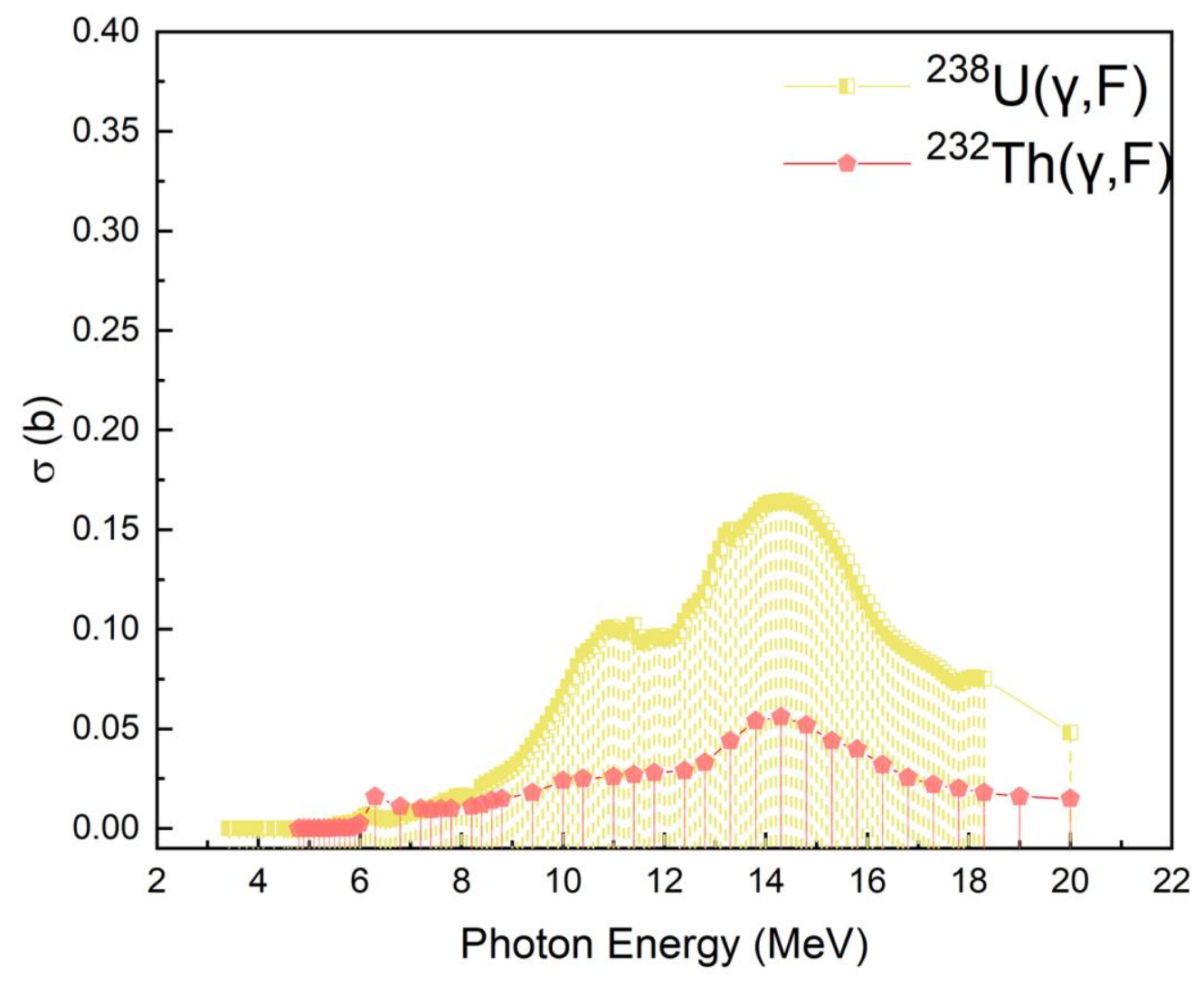

${ }^{1}$ ENDF-B/VII.0: Accessed from Janis 4.0, Nuclear Energy Agency, Organization for Economic Co-operation and Development. https://www.oecd-nea.org/janis/ 

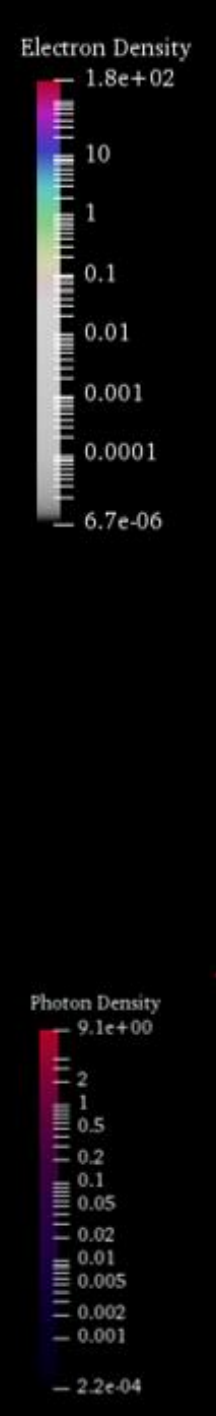

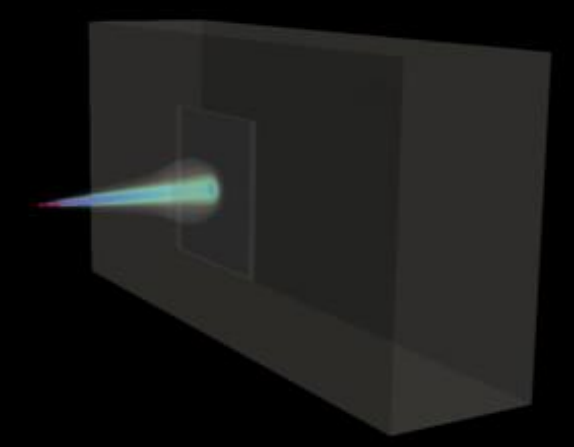

$8 \mathrm{MeV}$

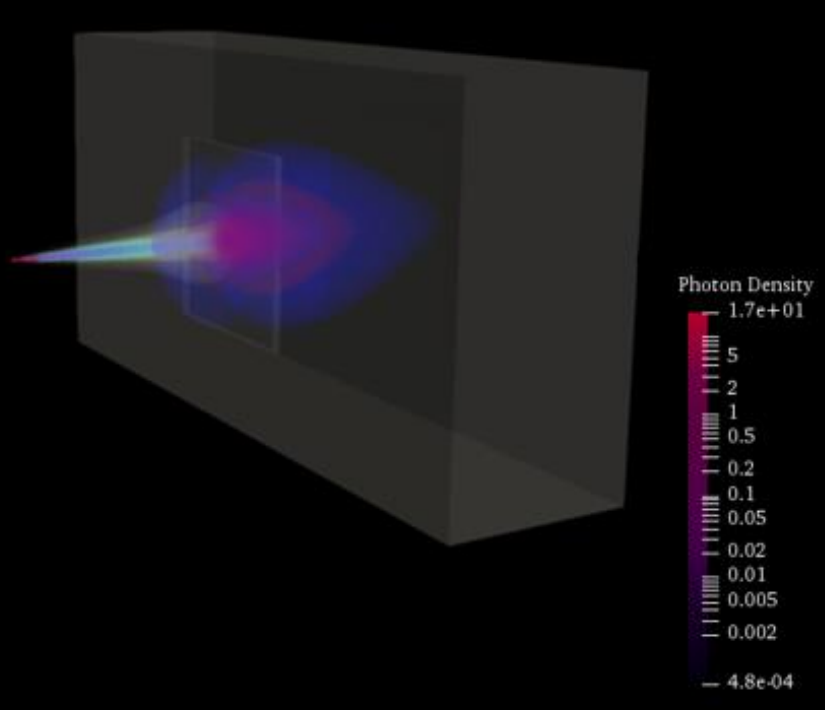

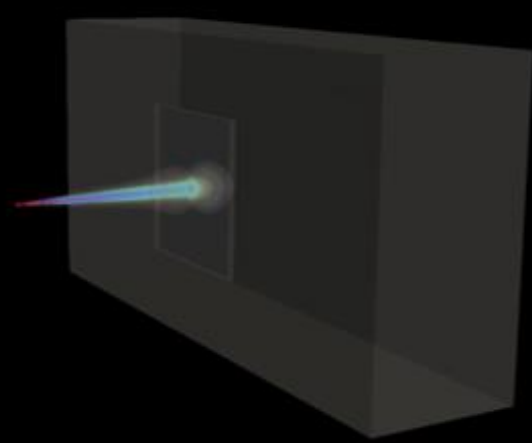

$14 \mathrm{MeV}$

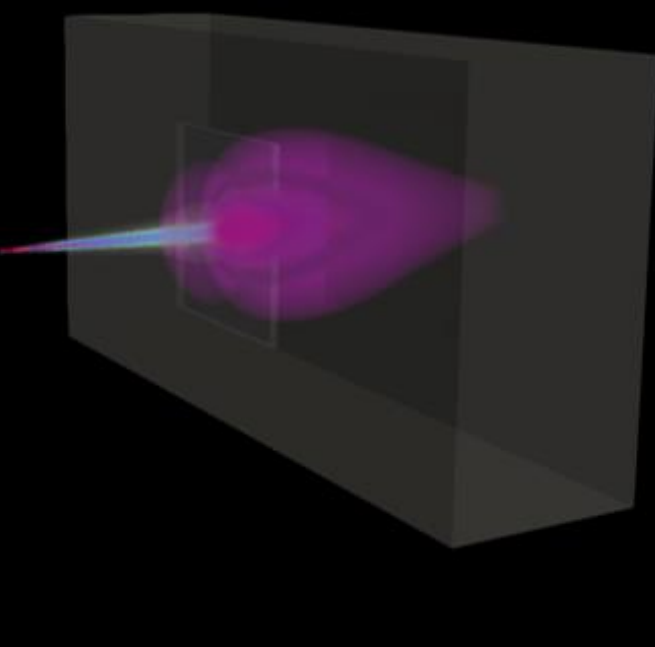

- MCNP6 3D mesh tally produced for electron and photon beam each E.E.

- Visualization of bremsstrahlung X-ray beam conversion

\section{$20 \mathrm{MeV}$}

How is yield effected

by an offset?
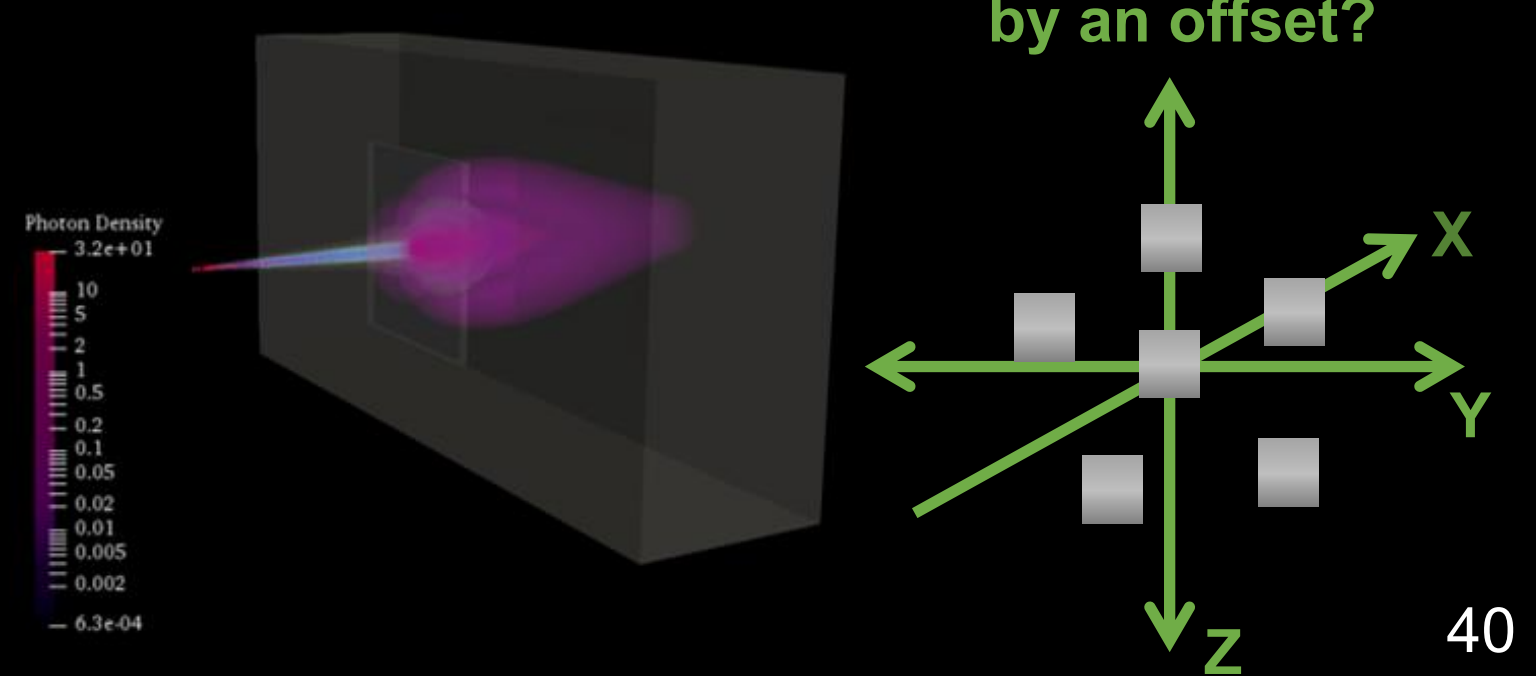


\section{Uncertainty Contribution from Target Offset}
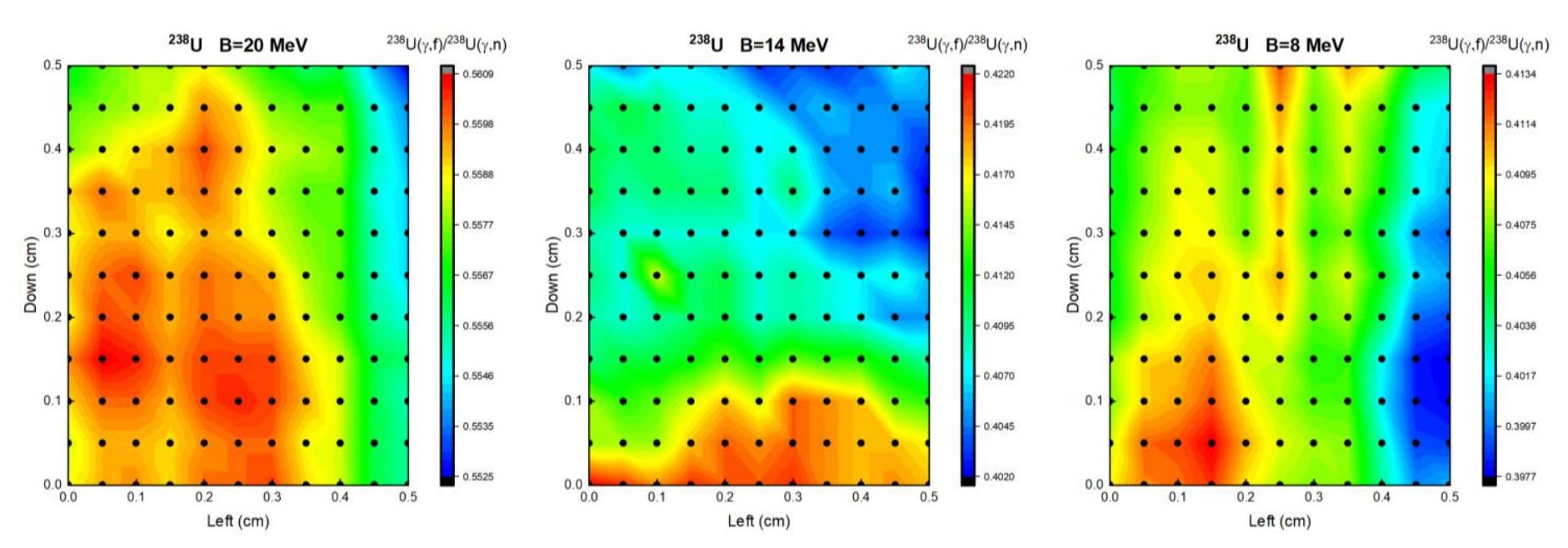

Ratio of ${ }^{238} \mathrm{U}$ photofission to ${ }^{237} \mathrm{U}$ production
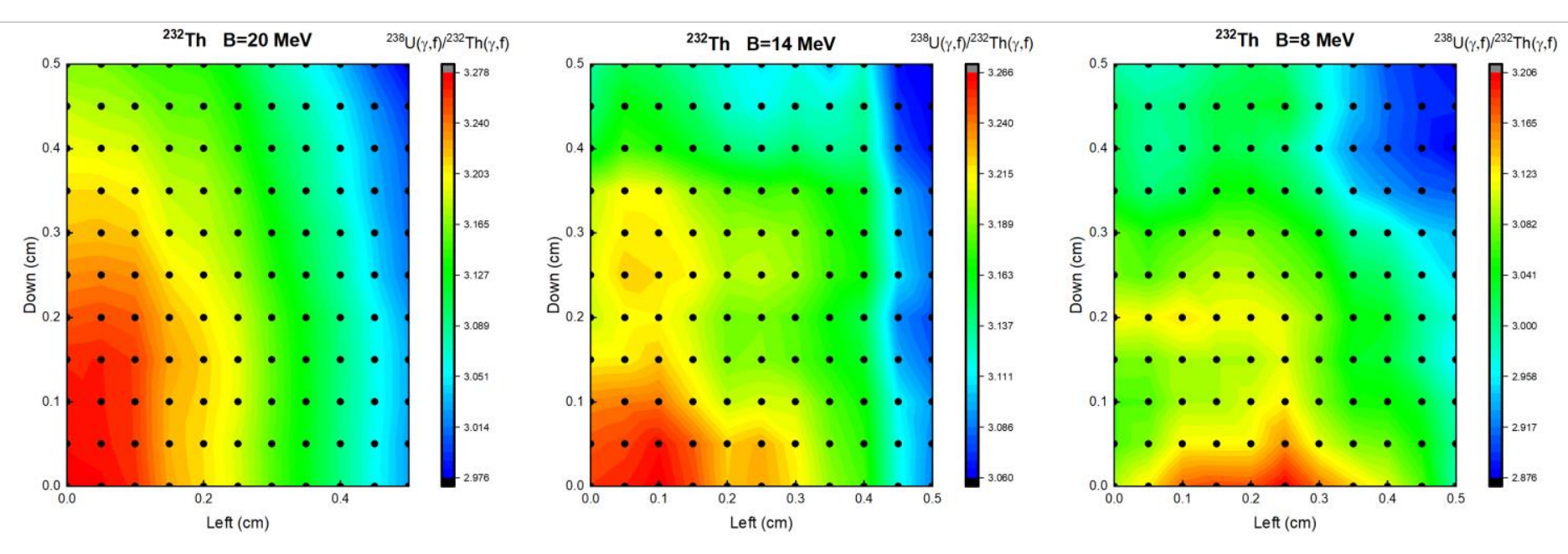

$$
R_{U 238 f}=\frac{\int_{0}^{B} \frac{\phi(B, E)}{d E} \sigma_{U 238, \gamma, f}(E) d E}{\int_{0}^{B} \frac{\phi(B, E)}{d E} \sigma_{U 238 \gamma, n}(E) d E}
$$

Ratio of ${ }^{238} \mathrm{U}$ photofission to ${ }^{232} \mathrm{Th}$ photofission

$$
R_{T h 238 f}=\frac{\int_{0}^{B} \frac{\phi(B, E)}{d E} \sigma_{U 238, \gamma, f}(E) d E}{\int_{0}^{B} \frac{\phi(B, E)}{d E} \sigma_{T h 232, \gamma, f}(E) d E}
$$




\section{Uncertainty Contribution from Target Offset}
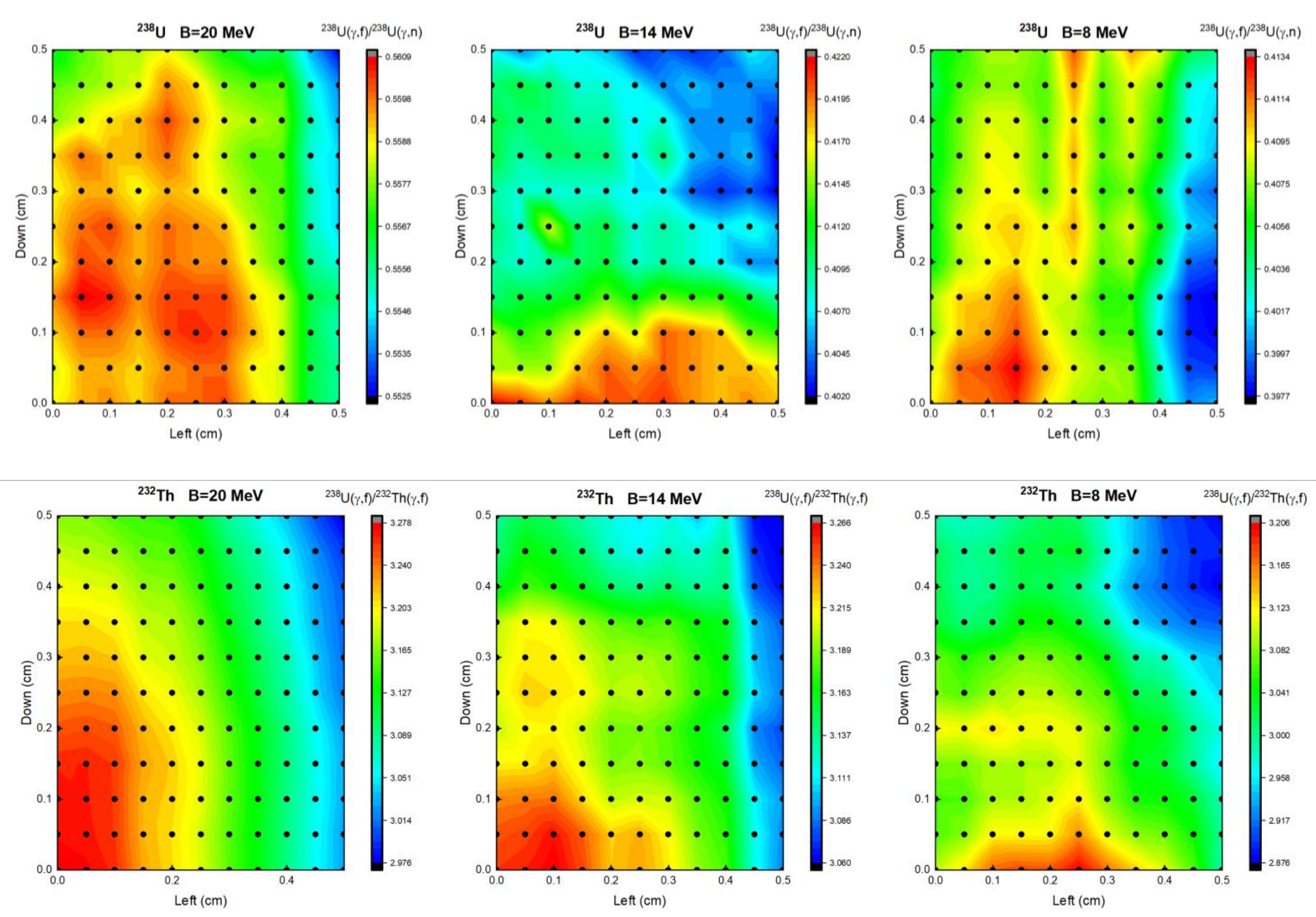

- 121 simulations for an offset of 0-0.5 $\mathrm{cm}$ in the left and down positions or each pair of target and endpoint energy

- Calculated incident beam energy ratio of cross sections

- Photofission to ${ }^{237} \mathrm{U}$ production for uranium irradiations

- Photofission of ${ }^{238} \mathrm{U}$ to ${ }^{232} \mathrm{Th}$ for thorium irradiations

- STD of sample taken as the conservative uncertainty of offset position during pneumatic transfer in calculating photofission events per pulse 